\title{
Article \\ Schur Complement-Based Infinity Norm Bounds for the Inverse of GDSDD Matrices
}

Yating Li and Yaqiang Wang *

check for updates

Citation: Li, Y.; Wang, Y. Schur Complement-Based Infinity Norm Bounds for the Inverse of GDSDD Matrices. Mathematics 2022, 10, 186. https://doi.org/10.3390/ math10020186

Academic Editor: Irina Cristea

Received: 29 November 2021

Accepted: 5 January 2022

Published: 7 January 2022

Publisher's Note: MDPI stays neutral with regard to jurisdictional claims in published maps and institutional affiliations.

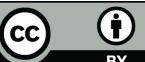

Copyright: () 2022 by the authors Licensee MDPI, Basel, Switzerland. This article is an open access article distributed under the terms and conditions of the Creative Commons Attribution (CC BY) license (https:// creativecommons.org/licenses/by/ $4.0 /)$.
School of Mathematics and Information Science, Baoji University of Arts and Sciences, Baoji 721013, China; yatingli820@163.com

* Correspondence: yaqiangwang1004@163.com

\begin{abstract}
Based on the Schur complement, some upper bounds for the infinity norm of the inverse of generalized doubly strictly diagonally dominant matrices are obtained. In addition, it is shown that the new bound improves the previous bounds. Numerical examples are given to illustrate our results. By using the infinity norm bound, a lower bound for the smallest singular value is given.
\end{abstract}

Keywords: GDSDD matrices; Schur complements; Infinity norm bounds; singular value

\section{Introduction}

Throughout the paper, let $n$ be an integer number, $N:=\{1,2, \ldots, n\}$ be the set of all indices, $C^{n \times n}$ (resp. $R^{n \times n}$ ) denote the set of all $n \times n$ complex (resp.real) matrices, $I \in R^{n \times n}$ be an identity matrix, $|A|=\left[\left|a_{i j}\right|\right] \in R^{n \times n}, r_{i}(A):=\sum_{j=1, j \neq i}^{j=n}\left|a_{i j}\right|$ denote deleted $i$ th row sum, and $J:=\left\{i \in N:\left|a_{i i}\right|>r_{i}(A)\right\}$.

Definition 1 ([1]). Let $A=\left[a_{i j}\right] \in \mathbb{C}^{n \times n}(n \geq 2)$, then $A$ is called:

(i) A row diagonally dominant (DD) if for all $i \in N$,

$$
\left|a_{i i}\right| \geq r_{i}(A)
$$

(ii) A strictly diagonally dominant (SDD) if all the strict inequalities in (1) hold;

(iii) A doubly strictly diagonally dominant (DSDD) matrix, if:

$$
\left|a_{i i}\right|\left|a_{j j}\right|>r_{i}(A) r_{j}(A), i, j \in N, i \neq j .
$$

The class of generalized doubly strictly diagonally dominant (GDSDD) matrices was presented by Gao and Wang in [2].

Definition 2 ([2]). A matrix $A$ is called a GDSDD matrix if $J \neq \varnothing$ and there exist proper subsets $N_{1}, N_{2}$ of $N$ such that $N_{1} \cap N_{2}=\varnothing, N_{1} \cup N_{2}=N$ and:

$$
\left(\left|a_{i i}\right|-\alpha_{i}\right)\left(\left|a_{j j}\right|-\beta_{j}\right)>\beta_{i} \alpha_{j}
$$

for any $i \in N_{1}$ and $j \in N_{2}$, where with $s=i$ or $j$,

$$
\alpha_{s}=\sum_{\substack{t \in N_{1} \\ t \neq s}}\left|a_{s t}\right|, \beta_{s}=\sum_{\substack{t \in N_{2} \\ t \neq s}}\left|a_{s t}\right|
$$

Here the $\alpha_{s}$ and $\beta_{s}$ may be interpreted as the sums of the absolute values of the nondiagonal elements in row $s$ that fall in the columns $N_{1}$ and $N_{2}$, respectively. When $n=2$, a GDSDD matrix is nothing but a $D S D D$ matrix. A matrix $A$ satisfying (3) may not 
be generalized doubly diagonally dominant for another pair subsets $N_{1}$ and $N_{2}$. Assuming that the matrix order is $n \geq 2$, we adopt the notation: $G D S D D_{n}^{N_{1}, N_{2}}$ for generalized doubly strictly diagonally dominant.

Definition 3 ([3]). A matrix $A$ is called an H-matrix if its comparison matrix $\mu(A)=\left[\mu_{i j}\right]$ defined by:

$$
\mu_{i j}=\left\{\begin{aligned}
\left|a_{i j}\right| & \text { if } i=j, \\
-\left|a_{i j}\right| & \text { if } i \neq j,
\end{aligned}\right.
$$

is an M-matrix, i.e., $(\mu(A))^{-1} \geq 0$.

It is shown in [3] that if $A$ is an $H$-matrix, then:

$$
(\mu(A))^{-1} \geq\left|A^{-1}\right| .
$$

In addition, it was shown that $S D D$ matrices, $D S D D$ matrices, and GDSDD matrices are subclass of $H$-matrices [4].

Upper bounds for the infinity norm of the inverse of nonsingular matrices can be used to the convergence analysis of matrix splitting and matrix multi-splitting iterative methods for solving the large sparse of linear equations. Moreover, the upper bounds can also be applied to the smallest singular value of matrices $[5,6]$.

One traditional method for finding upper bounds for the infinity norm of the inverse of nonsingular matrices is to use the definition and properties of a given matrix class; see [7-10] for details. The first work may due to Varah [10], who in 1975 presented the following upper bound for the infinity norm of the inverse for $S D D$ matrices.

Theorem 1 ([10]). If $A=\left[a_{i j}\right] \in C^{n \times n}$ is $S D D$, then:

$$
\left\|A^{-1}\right\|_{\infty} \leq \max _{i \in N} \frac{1}{\left|a_{i i}\right|-r_{i}(A)} .
$$

However, if the diagonal dominance of $A$ (i.e. $\left|a_{i i}\right|-r_{i}(A)$ ) is weak, Varah's bound may yield a large value. In 2020, based on the Schur complement, Li [11] obtained two upper bounds for the infinity norm of inverse of $S D D$ matrices.

Using the definition and properties of DSDD matrices, Liu, Zhang and Liu in [9] obtained an upper bound of $\left\|A^{-1}\right\|_{\infty}$ for a $D S D D$ matrix $A$ as follows.

Theorem 2 ([7]). If $A=\left[a_{i j}\right] \in C^{n \times n}$ is $D S D D$, then:

$$
\left\|A^{-1}\right\|_{\infty} \leq \max _{\substack{i, j \in N \\ i \neq j}} \frac{\left|a_{j j}\right|+r_{i}(A)}{\left|a_{i i}\right|\left|a_{j j}\right|-r_{i}(A) r_{j}(A)} .
$$

In 2020, based on the Schur complement, Sang [12] obtained two upper bounds for the infinity norm of $D S D D$ matrices.

And Moraa in [13] give an upper bound for the infinity norm of GDSDD matrices as follows.

Theorem 3. If $A=\left[a_{i j}\right] \in C^{n \times n}$ is $G D S D D_{n}^{N_{1}, N_{2}}$, then:

$$
\left\|A^{-1}\right\|_{\infty} \leq \max \left\{\max _{i \in N_{1}, j \in N_{2}} \frac{\left|a_{j j}\right|-\beta_{j}+\beta_{i}}{\left(\left|a_{i i}\right|-\alpha_{i}\right)\left(\left|a_{j j}\right|-\beta_{j}\right)-\alpha_{j} \beta_{i}}{ }^{\prime} \max _{i \in N_{1}, j \in N_{2}} \frac{\left|a_{i i}\right|-\alpha_{i}+\alpha_{j}}{\left(\left|a_{i i}\right|-\alpha_{i}\right)\left(\left|a_{j j}\right|-\beta_{j}\right)-\alpha_{j} \beta_{i}}\right\} .
$$


In this paper, based on the Schur complement, we present some upper bounds for the infinity norm of the inverse of GDSDD matrices, and numerical examples are given to show the effectiveness of the obtained results. In addition, applying these new bounds, a lower bound for the smallest singular value of GDSDD matrices is obtained.

\section{Schur Complement-Based Infinity Bounds for the Inverse of GDSDD Matrices}

Firstly, we recall the Schur complement of matrices.

Let $\alpha$ be a proper subset of $N$ and denote by $|\alpha|$ the cardinality of $\alpha$ and by $\alpha^{\prime}=N-\alpha$ the complement to $\alpha$ in $N$. For nonempty index sets $\alpha, \beta \subseteq N$, we write $A(\alpha, \beta)$ to mean the submatrix of $A \in C^{n \times n}$ lying in the rows indexed by $\alpha$ and the columns indexed by $\beta$. $A(\alpha, \alpha)$ is abbreviated to $A(\alpha)$. Throughout this paper, supposing $\alpha=\left\{i_{1}, i_{2}, \ldots, i_{l}\right\} \subset N$, the complement $\alpha^{\prime}=N-\alpha=\left\{j_{1}, j_{2}, \ldots, j_{m}\right\}$ and the elements of $\alpha$ and $\alpha^{\prime}$ are both conventionally arranged in increasing order. Furthermore, if $A(\alpha)$ is nonsingular, we define the Schur complement of $A$ with respect to $A(\alpha)$, which is denoted by $A / A(\alpha)$ or simply $A / \alpha$, to be:

$$
A / \alpha=A\left(\alpha^{\prime}\right)-A\left(\alpha^{\prime}, \alpha\right) A(\alpha)^{-1} A\left(\alpha, \alpha^{\prime}\right) .
$$

Liu, Huang, and Zhang in [14] proved that the Schur complements of GDSDD matrices are GDSDD matrices. Many similar or related results had been obtained; see $[9,15,16]$ for details.

For a given nonempty proper subset $\alpha$, there is always a permutation matrix $P$ such that:

$$
P^{\mathrm{T}} A P=\left[\begin{array}{cc}
A(\alpha) & A\left(\alpha, \alpha^{\prime}\right) \\
A\left(\alpha^{\prime}, \alpha\right) & A\left(\alpha^{\prime}\right)
\end{array}\right] .
$$

It is well known that the inverse of a permutation matrix is also a permutation matrix and the infinity norm for the inverse of a permutation matrix equals to $1\left(\|P\|_{\infty}=1\right)$. Hence, if $A$ is nonsingular, then:

$$
\left\|A^{-1}\right\|_{\infty}=\left\|P\left(P^{\mathrm{T}} A P\right)^{-1} P^{\mathrm{T}}\right\|_{\infty} \leq\left\|\left(\left(P^{\mathrm{T}} A P\right)^{-1}\right)\right\|_{\infty} .
$$

So we next only consider the upper bound for $\left\|\left(\left(P^{\mathrm{T}} A P\right)^{-1}\right)\right\|_{\infty}$. For the sake of simplicity, we consider that:

$$
P^{\mathrm{T}} A P=\left[\begin{array}{ll}
A_{11} & A_{12} \\
A_{21} & A_{22}
\end{array}\right]
$$

where $A_{11}=A(\alpha)$ and $A_{22}=A\left(\alpha^{\prime}\right)$.

Lemma 1 ([17]). Let $A \in C^{n \times n}$ with the form (6), and $I_{11}$ (resp. $I_{22}$ ) be the identity matrix of order $l$ (resp. $m$ ). If $A_{11}$ is nonsingular, then:

$$
S\left(P^{\mathrm{T}} A P\right) Q=\left[\begin{array}{cc}
A_{11} & 0 \\
0 & A_{22}-A_{21} A_{11}^{-1} A_{12}
\end{array}\right],
$$

where

$$
S=\left[\begin{array}{cc}
I_{11} & 0 \\
-A_{21} A_{11}^{-1} & I_{22}
\end{array}\right] \text { and } Q=\left[\begin{array}{cc}
I_{11} & -A_{11}^{-1} A_{12} \\
0 & I_{22}
\end{array}\right]
$$

From Lemma 1, we obtain:

$$
A=\left(P^{\mathrm{T}}\right)^{-1} S^{-1} S\left(P^{\mathrm{T}} A P\right) Q Q^{-1} P^{-1},
$$

and hence:

$$
A^{-1}=P Q\left(S\left(P^{\mathrm{T}} A P\right) Q\right)^{-1} S P^{\mathrm{T}},
$$


which implies that:

$$
\left\|A^{-1}\right\|_{\infty}=\left\|P Q\left(S P^{\mathrm{T}} A P Q\right)^{-1} S P^{\mathrm{T}}\right\|_{\infty} \leq\|Q\|_{\infty}\left\|\left(S P^{\mathrm{T}} A P Q\right)^{-1}\right\|_{\infty}\|S\|_{\infty} .
$$

This implies that if upper bounds for $\|S\|_{\infty},\|Q\|_{\infty}\left\|A_{11}^{-1}\right\|_{\infty^{\prime}}$ and $\left\|(A / \alpha)^{-1}\right\|_{\infty}$ are given, then the product of these bounds could be regarded as an upper bound for $\left\|A^{-1}\right\|_{\infty}$ by (7). It is not difficult to compute that:

$$
\begin{aligned}
& \|S\|_{\infty}=1+\left\|A_{21} A_{11}^{-1}\right\|_{\infty}, \\
& \|Q\|_{\infty}=1+\left\|A_{11}^{-1} A_{12}\right\|_{\infty},
\end{aligned}
$$

and

$$
\left\|\left(S P^{\mathrm{T}} A P Q\right)^{-1}\right\|_{\infty}=\max \left\{\left\|A_{11}^{-1}\right\|_{\infty}{ }_{\infty}\left\|\left(A_{22}-A_{21} A_{11}^{-1} A_{12}\right)^{-1}\right\|_{\infty}\right\} .
$$

In 2020, Li [11] gave an upper bound for $\|S\|_{\infty}$ as follows:

Lemma 2 ([11]). Let $A=\left[a_{i j}\right] \in C^{n \times n}$ be nonsingular with $a_{i i} \neq 0$ for $i \in N$, and $\varnothing \neq \alpha \subset N$. If $A_{11}$ is nonsingular, and:

$$
1>\max _{i \in \alpha} \frac{\max _{j \in \alpha, j \neq i}\left|a_{j i}\right|}{\left|a_{i i}\right|} \cdot(l-1),
$$

then

$$
\|S\|_{\infty} \leq \gamma(\alpha)=1+l \cdot \max _{i \in \alpha} \frac{\max _{j \in \alpha^{\prime}}\left|a_{j i}\right|}{\left|a_{i i}\right|} \cdot\left(1-\max _{i \in \alpha} \frac{\max _{j \in \alpha, j \neq i}\left|a_{j i}\right|}{\left|a_{i i}\right|} \cdot(l-1)\right)^{-1} .
$$

Next, we only consider upper bounds for $\|Q\|_{\infty}\left\|A_{11}^{-1}\right\|_{\infty}$ and $\left\|\left(A_{22}-A_{21} A_{11}^{-1} A_{12}\right)^{-1}\right\|_{\infty}$ when $A$ is $G D S D D_{n}^{N_{1}, N_{2}}$.

Lemma 3 ([14]). Let $A$ be $G D S D D_{n}^{N_{1}, N_{2}}$. Then $A\left(N_{1}\right)$ and $A\left(N_{2}\right)$ are SDD.

Lemma 4 ([14]). Let $A$ be GDSDD $D_{n}^{N_{1}, N_{2}}$. Then $N_{1} \subseteq$ J or $N_{2} \subseteq J$.

In this paper, we assume $N_{1} \subseteq J$.

Lemma 5 ([14]). Let $A$ be $G D S D D_{n}^{N_{1}, N_{2}}$ and $\alpha \subset N$. Then $A(\alpha)$ is $G D S D D_{|\alpha|}^{N_{1} \cap \alpha_{1} N_{2} \cap \alpha}$.

Lemma 6 ([14]). Let $A$ be GDSDD $D_{n}^{N_{1}, N_{2}}$. Then for any proper subset $\alpha$ of $N, A / \alpha$ is $G D S D D_{|N-\alpha|}^{N_{1}-\alpha, N_{2}-\alpha}$.

Lemma 7 ([18]). Let $A=\left[a_{i j}\right] \in C^{n \times n}$ be $S D D$, and $B=\left[b_{i j}\right] \in C^{n \times m}$. Then:

$$
\left\|A^{-1} B\right\|_{\infty} \leq \max _{i \in N} \frac{R_{i}(B)}{\left|a_{i i}\right|-r_{i}(A)}
$$

where $R_{i}(B)=\sum_{k=1}^{m}\left|b_{i k}\right|$ 
Lemma 8. Let $A=\left[a_{i j}\right] \in C^{n \times n}$ be $G D S D D_{n}^{N_{1}, N_{2}}$, and $B=\left[b_{i j}\right] \in C^{n \times m}$. Then: $\left\|A^{-1} B\right\|_{\infty} \leq \max \left\{\max _{\substack{i \in N_{1} \\ j \in N_{2}}} \frac{\left(\left|a_{j j}\right|-\beta_{j}\right) R_{i}(B)+\beta_{i} R_{j}(B)}{\left(\mid \alpha_{i}\right)\left(\left|a_{j j}\right|-\beta_{j}\right)-\beta_{i} \alpha_{j}}, \max _{\substack{i \in N_{1} \\ j \in N_{2}}} \frac{\left.\left(\left|a_{i i}\right|-\alpha_{i i}\right) R_{j}(B)+\alpha_{i}\right)\left(\left|a_{j j}\right|-\beta_{j} R_{i}(B)-\beta_{i} \alpha_{j}\right.}{\left(\mid a_{j}\right.}\right\}$, where $R_{i}(B)=\sum_{k=1}^{m}\left|b_{i k}\right|$.

Proof. Since there exists an $m$-dimensional vector $x=\left(x_{1}, x_{2}, \ldots, x_{m}\right)^{\mathrm{T}}$ such that $\|x\|_{\infty}=1$ and:

$$
\left\|A^{-1} B\right\|_{\infty}=\left\|A^{-1} B x\right\|_{\infty}=\|y\|_{\infty}=\max _{i \in N}\left|y_{i}\right|
$$

where $y=A^{-1} B x=\left(y_{1}, y_{2}, \ldots, y_{n}\right)^{\mathrm{T}}$, we have $A y=B x$, and let $\left|y_{p}\right|=\max _{i \in N_{1}}\left|y_{i}\right|,\left|y_{q}\right|=$ $\max _{j \in N_{2}}\left|y_{j}\right|$, which implies that:

$$
a_{i i} y_{i}+\sum_{\substack{j \in N \\ j \neq i}} a_{i j} y_{j}=\sum_{k=1}^{m} b_{i k} x_{k}, i \in N
$$

Then,

$$
\begin{gathered}
\sum_{k=1}^{m} b_{p k} x_{k}=a_{p p} y_{p}+\sum_{\substack{j \in N \\
j \neq p}} a_{p j} y_{j}=a_{p p} y_{p}+\sum_{\substack{j \in N_{1} \\
j \neq p}} a_{p j} y_{j}+\sum_{j \in N_{2}} a_{p j} y_{j}, \\
\sum_{k=1}^{m} b_{q k} x_{k}=a_{q q} y_{q}+\sum_{\substack{j \in N \\
j \neq q}} a_{q j} y_{j}=a_{q q} y_{q}+\sum_{j \in N_{1}} a_{q j} y_{j}+\sum_{j \in N_{2}, j \neq q} a_{q j} y_{j} .
\end{gathered}
$$

We can obtain:

$$
\begin{aligned}
\sum_{k=1}^{m}\left|b_{p k}\right| \max _{1 \leq k \leq m}\left|x_{k}\right| & \geq \sum_{k=1}^{m}\left|b_{p k}\right|\left|x_{k}\right| \geq\left|\sum_{k=1}^{m} b_{p k} x_{k}\right| \\
& =\left|a_{p p} y_{p}+\sum_{\substack{j \in N \\
j \neq p}} a_{p j} y_{j}\right| \\
& =\left|a_{p p} y_{p}+\sum_{\substack{j \in N_{1} \\
j \neq p}} a_{p j} y_{j}+\sum_{j \in N_{2}} a_{p j} y_{j}\right| \\
& \geq\left|a_{p p} y_{p}\right|-\left|\sum_{\substack{j \in N_{1} \\
j \neq p}} a_{p j} y_{j}\right|-\left|\sum_{j \in N_{2}} a_{p j} y_{j}\right| \\
& \geq\left|a_{p p}\right|\left|y_{p}\right|-\sum_{\substack{j \in N_{1} \\
j \neq p}}\left|a_{p j}\right|\left|y_{j}\right|-\sum_{j \in N_{2}}\left|a_{p j}\right|\left|y_{j}\right| \\
& \geq\left|a_{p p}\right|\left|y_{p}\right|-\sum_{\substack{j \in N_{1} \\
j \neq p}}\left|a_{p j}\right|\left|y_{p}\right|-\sum_{j \in N_{2}}\left|a_{p j}\right|\left|y_{q}\right| \\
& =\left|a_{p p}\right|\left|y_{p}\right|-\alpha_{p}\left|y_{p}\right|-\beta_{p}\left|y_{q}\right|
\end{aligned}
$$

and

$$
\begin{aligned}
\sum_{k=1}^{m}\left|b_{q k}\right| \max _{1 \leq k \leq m}\left|x_{k}\right| & \geq \sum_{k=1}^{m}\left|b_{q k}\right|\left|x_{k}\right| \geq\left|\sum_{k=1}^{m} b_{q k} x_{k}\right| \\
& =\left|a_{q q} y_{q}+\sum_{\substack{j \in N \\
j \neq q}} a_{q j} y_{j}\right|
\end{aligned}
$$




$$
\begin{aligned}
& =\left|a_{q q} y_{q}+\sum_{j \in N_{1}} a_{q j} y_{j}+\sum_{\substack{j \in N_{2} \\
j \neq q}} a_{q j} y_{j}\right| \\
& \geq\left|a_{q q} y_{q}\right|-\left|\sum_{j \in N_{1}} a_{q j} y_{j}\right|-\left|\sum_{\substack{j \in N_{2} \\
j \neq q}} a_{q j} y_{j}\right| \\
& \geq\left|a_{q q}\right|\left|y_{q}\right|-\sum_{j \in N_{1}}\left|a_{q j}\right|\left|y_{j}\right|-\sum_{\substack{j \in N_{2} \\
j \neq q}}\left|a_{q j}\right|\left|y_{j}\right| \\
& \geq\left|a_{q q}\right|\left|y_{q}\right|-\sum_{j \in N_{1}}\left|a_{q j}\right|\left|y_{p}\right|-\sum_{\substack{j \in N_{2} \\
j \neq q}}\left|a_{q j}\right|\left|y_{q}\right| \\
& =\left|a_{q q}\right|\left|y_{p}\right|-\alpha_{q}\left|y_{p}\right|-\beta_{q}\left|y_{q}\right| .
\end{aligned}
$$

That is to say,

$$
\sum_{k=1}^{m}\left|b_{p k}\right| \max _{1 \leq k \leq m}\left|x_{k}\right| \geq\left|a_{p p}\right|\left|y_{p}\right|-\alpha_{p}\left|y_{p}\right|-\beta_{p}\left|y_{q}\right|
$$

and

$$
\sum_{k=1}^{m}\left|b_{q k}\right| \max _{1 \leq k \leq m}\left|x_{k}\right| \geq\left|a_{q q}\right|\left|y_{p}\right|-\alpha_{q}\left|y_{p}\right|-\beta_{q}\left|y_{q}\right|
$$

The proof is divided into two cases.

Case 1: $\left|y_{p}\right| \geq\left|y_{q}\right|$. Then, $(12) \times\left(\left|a_{q q}\right|-\beta_{q}\right)+(13) \times \beta_{p}$ yields

$\left(\left|a_{q q}\right|-\beta_{q}\right) \sum_{k=1}^{m}\left|b_{p k}\right| \max _{1 \leq k \leq m}\left|x_{k}\right|+\beta_{p} \sum_{k=1}^{m}\left|b_{q k}\right| \max _{1 \leq k \leq m}\left|x_{k}\right| \geq\left|y_{p}\right|\left[\left(\left|a_{p p}\right|-\alpha_{p}\right)\left(\left|a_{q q}\right|-\beta_{q}\right)-\beta_{p} \alpha_{q}\right]$,

which leads to:

$$
\begin{aligned}
\left\|A^{-1} B\right\|_{\infty} & =\max _{i \in N}\left|y_{i}\right|=\left|y_{p}\right| \\
\leq & \frac{\left(\left|a_{q q}\right|-\beta_{q}\right) \sum_{k=1}^{m}\left|b_{p k}\right| \max _{1 \leq k \leq m}\left|x_{k}\right|+\beta_{p} \sum_{k=1}^{m}\left|b_{q k}\right| \max _{1 \leq k \leq m}\left|x_{k}\right|}{\left(\left|a_{p p}\right|-\alpha_{p}\right)\left(\left|a_{q q}\right|-\beta_{q}\right)-\alpha_{q} \beta_{p}} \\
= & \frac{\left(\left|a_{q q}\right|-\beta_{q}\right) \sum_{k=1}^{m}\left|b_{p k}\right|+\beta_{p} \sum_{k=1}^{m}\left|b_{q k}\right|}{\left(\left|a_{p p}\right|-\alpha_{p}\right)\left(\left|a_{q q}\right|-\beta_{q}\right)-\alpha_{q} \beta_{p}} \\
\leq & \max _{i \in N_{1}, j \in N_{2}} \frac{\left(\left|a_{j j}\right|-\beta_{j}\right) \sum_{k=1}^{m}\left|b_{i k}\right|+\beta_{i} \sum_{k=1}^{m}\left|b_{j k}\right|}{\left(\left|a_{i i}\right|-\alpha_{i}\right)\left(\left|a_{j j}\right|-\beta_{j}\right)-\alpha_{j} \beta_{i}} \\
& =\max _{i \in N_{1}, j \in N_{2}} \frac{\left(\left|a_{j j}\right|-\beta_{j}\right) R_{i}(B)+\beta_{i} R_{j}(B)}{\left(\left|a_{i i}\right|-\alpha_{i}\right)\left(\left|a_{j j}\right|-\beta_{j}\right)-\alpha_{j} \beta_{i}} .
\end{aligned}
$$

Case 2: $\left|y_{p}\right|<\left|y_{q}\right|$. Then, $(12) \times \alpha_{q}+(13) \times\left(\left|a_{p p}\right|-\alpha_{p}\right)$ yields:

$\alpha_{q} \sum_{k=1}^{m}\left|b_{p k}\right| \max _{1 \leq k \leq m}\left|x_{k}\right|+\left(\left|a_{p p}\right|-\alpha_{p}\right) \sum_{k=1}^{m}\left|b_{q k}\right| \max _{1 \leq k \leq m}\left|x_{k}\right| \geq\left|y_{q}\right|\left[\left(\left|a_{p p}\right|-\alpha_{p}\right)\left(\left|a_{q q}\right|-\beta_{q}\right)-\beta_{p} \alpha_{q}\right]$,

which leads to: 


$$
\begin{aligned}
\left\|A^{-1} B\right\|_{\infty} & =\max _{i \in N}\left|y_{i}\right|=\left|y_{q}\right| \\
& \leq \frac{\alpha_{q} \sum_{k=1}^{m}\left|b_{p k}\right| \max _{1 \leq k \leq m}\left|x_{k}\right|+\left(\left|a_{p p}\right|-\alpha_{p}\right) \sum_{k=1}^{m}\left|b_{q k}\right| \max _{1 \leq k \leq m}\left|x_{k}\right|}{\left(\left|a_{p p}\right|-\alpha_{p}\right)\left(\left|a_{q q}\right|-\beta_{q}\right)-\alpha_{q} \beta_{p}} \\
& =\frac{\alpha_{q} \sum_{k=1}^{m}\left|b_{p k}\right|+\left(\left|a_{p p}\right|-\alpha_{p}\right) \sum_{k=1}^{m}\left|b_{q k}\right|}{\left(\left|a_{p p}\right|-\alpha_{p}\right)\left(\left|a_{q q}\right|-\beta_{q}\right)-\alpha_{q} \beta_{p}} \\
& \leq \max _{i \in N_{1}, j \in N_{2}} \frac{\alpha_{j} \sum_{k=1}^{m}\left|b_{i k}\right|+\left(\left|a_{i i}\right|-\alpha_{i}\right) \sum_{k=1}^{m}\left|b_{j k}\right|}{\left(\left|a_{i i}\right|-\alpha_{i}\right)\left(\left|a_{j j}\right|-\beta_{j}\right)-\alpha_{j} \beta_{i}} \\
& =\max _{i \in N_{1}, j \in N_{2}} \frac{\left(\left|a_{i i}\right|-\alpha_{i}\right) R_{j}(B)+\alpha_{j} R_{i}(B)}{\left(\left|a_{i i}\right|-\alpha_{i}\right)\left(\left|a_{j j}\right|-\beta_{j}\right)-\alpha_{j} \beta_{i}} .
\end{aligned}
$$

The conclusion follows from equality (11) and inequalities (14) and (15).

Corollary 1. Let $A=\left[a_{i j}\right] \in C^{n \times n}$ be GDSDD $D_{n}^{N_{1}, N_{2}}$, and $\varnothing \neq \alpha \subset N$. Then:

$$
\|Q\|_{\infty}=1+\left\|A_{11}^{-1} A_{12}\right\|_{\infty} \leq 1+\varphi(\alpha),
$$

where

$$
\varphi(\alpha)=\max \left\{\max _{\substack{i \in N_{1} \cap \alpha \\ j \in N_{2} \cap \alpha}} \frac{\left(\left|a_{j j}\right|-\beta_{j}\right) R_{i}\left(A_{12}\right)+\beta_{i} R_{j}\left(A_{12}\right)}{\left(\left|a_{i i}\right|-\alpha_{i}\right)\left(\left|a_{j j}\right|-\beta_{j}\right)-\beta_{i} \alpha_{j}} \max _{\substack{i \in N_{1} \cap \alpha \\ j \in N_{2} \cap \alpha}} \frac{\left(\left|a_{i i}\right|-\alpha_{i}\right) R_{j}\left(A_{12}\right)+\alpha_{j} R_{i}\left(A_{12}\right)}{\left(\left|a_{i i}\right|-\alpha_{i}\right)\left(\left|a_{j j}\right|-\beta_{j}\right)-\beta_{i} \alpha_{j}}\right\} .
$$

Specially, if $\alpha \subset N_{1}$ or $\alpha \subset N_{2}$, then $A_{11}$ is $S D D$, and

$$
\|Q\|_{\infty}=1+\left\|A_{11}^{-1} A_{12}\right\|_{\infty} \leq 1+\max _{i \in \alpha} \frac{R_{i}\left(A_{12}\right)}{\left|a_{i i}\right|-r_{i}\left(A_{11}\right)} .
$$

For the sake of convenience, denote:

$$
\begin{gathered}
{[l]=\{1,2, \ldots, l\},} \\
{[m]=\{1,2, \ldots, m\},} \\
v_{j_{k}}:=\left(a_{j_{k} i_{1}}, \ldots, a_{j_{k} i_{l}}\right), \\
w_{j_{k}}:=\left(a_{i_{1} j_{k}}, \ldots, a_{i_{l_{j}} j_{k}}\right)^{\mathrm{T}}, \\
\left|v_{j_{k}}\right|:=\left(\left|a_{j_{k} i_{1}}\right|, \ldots,\left|a_{j_{k} i_{l}}\right|\right), \\
\left|w_{j_{k}}\right|:=\left(\left|a_{i_{1} j_{k}}\right|, \ldots,\left|a_{i_{l} j_{k}}\right|\right)^{\mathrm{T}},
\end{gathered}
$$

for any $k=1,2, \ldots, m$. And thus:

$$
\sum_{k \in[m]}\left|w_{j_{k}}\right|=\left(\sum_{k \in[m]}\left|a_{i_{1} j_{k}}\right|, \ldots, \sum_{k \in[m]}\left|a_{i_{l} j_{k}}\right|\right)^{\mathrm{T}}=\sum_{j_{k} \in \alpha^{\prime}}\left|w_{j_{k}}\right| .
$$

Lemma 9. Let $x=\{\mu[A(\alpha)]\}^{-1} \sum_{k \in[m]}\left|w_{j_{k}}\right|=\left(x_{1}, \ldots, x_{l}\right)^{\mathrm{T}}, x_{g}=\max _{k \in[l]} x_{k}$. If $i_{g} \in \alpha$ is a strictly diagonally dominant row, then:

$$
0 \leq x_{k}<1, k \in[l] .
$$


Proof.

$$
x=\{\mu[A(\alpha)]\}^{-1} \sum_{k \in[m]}\left|w_{j_{k}}\right|=\left(x_{1}, \ldots, x_{l}\right)^{\mathrm{T}}, \text { i.e., } \mu[A(\alpha)] x=\sum_{k \in[m]}\left|w_{j_{k}}\right| .
$$

For any $\alpha \subset N, A_{11}$ is GDSDD, so is $\mu(A(\alpha))$, and consequently, $\mu(A(\alpha))$ is an $H$ matrix, and $\{\mu[A(\alpha)]\}^{-1} \geq 0$ by (4). Hence, $x$ is nonnegative. Let $x_{g}=\max _{k \in[l]} x_{k}$. By $i_{g}$ is a strictly diagonally dominant row, then:

$$
\left|a_{i_{g} i_{g}}\right|>\sum_{v \in[l], v \neq g}\left|a_{i_{g} i_{v}}\right|+\sum_{k \in[m]}\left|a_{i_{g} j_{k}}\right| .
$$

By the $g$ th equality of (20) and inequality (21), we have:

$$
\sum_{k \in[m]}\left|a_{i_{g} j_{k}}\right|=\left|a_{i_{g} i_{g}}\right| x_{g}-\sum_{v \in[l], v \neq g}\left|a_{i_{g} i_{v}}\right| x_{v} \geq x_{g}\left(\left|a_{i_{g} i_{g}}\right|-\sum_{v \in[l], v \neq g}\left|a_{i_{g} i_{v}}\right|\right),
$$

and hence:

$$
x_{g} \leq \frac{\sum_{k \in[m]}\left|a_{i_{g} j_{k}}\right|}{\left|a_{i_{g} i_{g}}\right|-\sum_{v \in[l], v \neq g}\left|a_{i_{g} i_{v}}\right|}<1
$$

which implies that:

$$
0 \leq x_{k}<1, k \in[l] .
$$

The proof is completed.

Theorem 4. Let $A=\left[a_{i j}\right]$ be GDSDD $D_{n}^{N_{1}, N_{2}}$ with $a_{i i} \neq 0, i \in N$, and $\varnothing \neq \alpha \subset N$. Assume that (8) holds, if $\alpha \subset N_{1}$, then:

$$
\left\|A^{-1}\right\|_{\infty} \leq \gamma(\alpha) \cdot\left(1+\max _{i \in \alpha} \frac{R_{i}\left(A_{12}\right)}{\left|a_{i i}\right|-r_{i}\left(A_{11}\right)}\right) \cdot \theta(\alpha),
$$

where

$$
\begin{gathered}
\theta(\alpha)=\max \left\{\max _{i \in \alpha} \frac{1}{\left|a_{i i}\right|-r_{i}\left(A_{11}\right)}, \eta(\alpha)\right\}, \\
\eta(\alpha)=\max \left\{\max _{\substack{i, j \in \alpha^{\prime} \\
i \in N_{1}-\alpha, j \in N_{2}}} \frac{\left|a_{j j}\right|-\beta_{j}(A)+\beta_{i}(A)+\sum_{k \in \alpha}\left|a_{j k}\right|+\sum_{k \in \alpha}\left|a_{i k}\right|}{y_{i, j}-z_{i, j}},\right. \\
\left.\max _{\substack{i, j \in \alpha^{\prime} \\
i \in N_{1}-\alpha, j \in N_{2}}} \frac{\left|a_{i i}\right|+\alpha_{j}(A)-\sum_{k \in N_{1}-\alpha}\left|a_{i k}\right|+\sum_{k \in \alpha}\left|a_{i k}\right|}{y_{i, j}-z_{i, j}}\right\},
\end{gathered}
$$

$$
\begin{gathered}
y_{i, j}=\left(\left|a_{i i}\right|-\sum_{k \in N_{1}-\alpha}\left|a_{i k}\right|-\left|v_{i}\right|\{\mu[A(\alpha)]\}^{-1} \sum_{k \in N_{1}-\alpha}\left|w_{k}\right|\right)\left(\left|a_{j j}\right|-\beta_{j}(A)-\left|v_{j}\right|\{\mu[A(\alpha)]\}^{-1} \sum_{k \in N_{2}}\left|w_{k}\right|\right), \\
z_{i, j}=\left(\sum_{k \in N_{1}-\alpha}\left|a_{j k}\right|+\left|v_{j}\right|\{\mu[A(\alpha)]\}^{-1} \sum_{k \in N_{1}-\alpha}\left|w_{k}\right|\right)\left(\beta_{i}(A)+\left|v_{i}\right|\{\mu[A(\alpha)]\}^{-1} \sum_{k \in N_{2}}\left|w_{k}\right|\right) .
\end{gathered}
$$

Proof. Denote the $(s, t)$-entry of $A / \alpha$ by $\left(a_{s t}^{\prime}\right)$. Then for any $s, t \in[m]$, 


$$
a_{s t}^{\prime}=a_{j_{s} j_{t}}-v_{j_{s}}[A(\alpha)]^{-1} w_{j_{t}}
$$

and by inequality (4), we have:

$$
\begin{aligned}
\left|a_{s t}^{\prime}\right| & =\left|a_{j_{s} j_{t}}-v_{j_{s}}[A(\alpha)]^{-1} w_{j_{t}}\right| \\
& \leq\left|a_{j_{s} j_{t}}\right|+\left|v_{j_{s}}\right||[A(\alpha)]|^{-1}\left|w_{j_{t}}\right| \\
& \leq\left|a_{j_{s} j_{t}}\right|+\left|v_{j_{s}}\right|\{\mu[A(\alpha)]\}^{-1}\left|w_{j_{t}}\right|, \\
\left|a_{s t}^{\prime}\right| & =\left|a_{j_{s} j_{t}}-v_{j_{s}}[A(\alpha)]^{-1} w_{j_{t}}\right| \\
& \geq\left|a_{j_{s} j_{t}}\right|-\left|v_{j_{s}}\right||[A(\alpha)]|^{-1}\left|w_{j_{t}}\right| \\
& \geq\left|a_{j_{s} j_{t}}\right|-\left|v_{j_{s}}\right|\{\mu[A(\alpha)]\}^{-1}\left|w_{j_{t}}\right| .
\end{aligned}
$$

Since $A$ is $G D S D D_{n}^{N_{1}, N_{2}}, \alpha \subset N_{1}$, by Lemmas 5 and 6 , we have $A_{11}$ is $S D D$, and $A / \alpha$ is $G D S D D_{|N-\alpha|}^{N_{1}-\alpha, N_{2}-\alpha}$. Applying the Varah's bound to $A_{11}$, we get:

$$
\left\|A_{11}^{-1}\right\|_{\infty} \leq \max _{i \in \alpha} \frac{1}{\left|a_{i i}\right|-r_{i}\left(A_{11}\right)} .
$$

Applying Theorem 3 to $A$ / $\alpha$ yields:

$$
\begin{aligned}
\left\|(A / \alpha)^{-1}\right\|_{\infty} \leq \max \left\{\max _{\substack{s, t \in[m] \\
j_{s} \in N_{1}-\alpha, j_{t} \in N_{2}}} \frac{\left|a_{t t}^{\prime}\right|-\beta_{t}(A / \alpha)+\beta_{s}(A / \alpha)}{\left(\left|a_{S S}^{\prime}\right|-\alpha_{s}(A / \alpha)\right)\left(\left|a_{t t}^{\prime}\right|-\beta_{t}(A / \alpha)\right)-\alpha_{t}(A / \alpha) \beta_{s}(A / \alpha)},\right. \\
\left.\max _{\substack{s, t \in[m] \\
j_{s} \in N_{1}-\alpha, j_{t} \in N_{2}}} \frac{\left|a_{S S}^{\prime}\right|-\alpha_{s}(A / \alpha)+\alpha_{t}(A / \alpha)}{\left(\left|a_{S S}^{\prime}\right|-\alpha_{S}(A / \alpha)\right)\left(\left|a_{t t}^{\prime}\right|-\beta_{t}(A / \alpha)\right)-\alpha_{t}(A / \alpha) \beta_{s}(A / \alpha)}\right\} .
\end{aligned}
$$

By Theorem 2 in [14], we have:

$$
\begin{aligned}
& \left(\left|a_{s s}^{\prime}\right|-\alpha_{s}(A / \alpha)\right)\left(\left|a_{t t}^{\prime}\right|-\beta_{t}(A / \alpha)\right)-\alpha_{t}(A / \alpha) \beta_{s}(A / \alpha) \\
& \geq\left(\left|a_{j_{s} j_{s}}\right|-\sum_{\substack{j_{k} \in N_{1}-\alpha \\
k \neq s}}\left|a_{j_{s} j_{k}}\right|-\left|v_{j_{s}}\right|\{\mu[A(\alpha)]\}^{-1} \sum_{j_{k} \in N_{1}-\alpha}\left|w_{j_{k}}\right|\right)\left(\left|a_{j_{t} j_{t}}\right|-\sum_{\substack{j_{k} \in N_{2} \\
k \neq t}}\left|a_{j_{t} j_{k}}\right|\right. \\
& \left.-\left|v_{j_{t}}\right|\{\mu[A(\alpha)]\}^{-1} \sum_{j_{k} \in N_{2}}\left|w_{j_{k}}\right|\right)-\left(\sum_{j_{k} \in N_{1}-\alpha}\left|a_{j_{t} j_{k}}\right|+\left|v_{j_{t}}\right|\{\mu[A(\alpha)]\}^{-1} \sum_{\substack{j_{k} \in N_{1}-\alpha \\
j_{k}}}\left|w_{j_{k}}\right|\right)\left(\sum_{j_{k} \in N_{2}}\left|a_{j_{s} j_{k}}\right|\right. \\
& \left.+\left|v_{j_{s}}\right|\{\mu[A(\alpha)]\}^{-1} \sum_{j_{k} \in N_{2}}\left|w_{j_{k}}\right|\right)
\end{aligned}
$$$$
>0 \text {. }
$$

Now, the upper bounds of $\left|a_{t t}^{\prime}\right|-\beta_{t}(A / \alpha)+\beta_{s}(A / \alpha)$ and $\left|a_{s s}^{\prime}\right|-\alpha_{s}(A / \alpha)+\alpha_{t}(A / \alpha)$ are considered. Since $\alpha \subset N_{1} \subseteq J$, it satisfies Lemma 9, which implies that (19) holds.

By (19), (20), (23) and (24), we have:

$$
\begin{aligned}
& \left|a_{t t}^{\prime}\right|-\beta_{t}(A / \alpha)+\beta_{s}(A / \alpha) \\
& =\left|a_{t t}^{\prime}\right|-\sum_{\substack{j_{k} \in N_{2} \\
k \neq t}}\left|a_{t k}^{\prime}\right|+\sum_{j_{k} \in N_{2}}\left|a_{s k}^{\prime}\right| \\
& \leq\left|a_{j_{t} j_{t}}\right|+\left|v_{j_{t}}\right|\{\mu[A(\alpha)]\}^{-1}\left|w_{j_{t}}\right|-\sum_{\substack{j_{k} \in N_{2} \\
k \neq t}}\left(\left|a_{j_{t} j_{k}}\right|-\left|v_{j_{t}}\right|\{\mu[A(\alpha)]\}^{-1}\left|w_{j_{k}}\right|\right) \\
& +\sum_{j_{k} \in N_{2}}\left(\left|a_{j_{s} j_{k}}\right|+\left|v_{j_{s}}\right|\{\mu[A(\alpha)]\}^{-1}\left|w_{j_{k}}\right|\right) \quad \text { (by inequalities (23) and (24)) } \\
& =\left|a_{j_{t} j_{t}}\right|+\left|v_{j_{t}}\right|\{\mu[A(\alpha)]\}^{-1}\left|w_{j_{t}}\right|-\sum_{\substack{j_{k} \in N_{2} \\
k \neq t}}\left|a_{j_{t} j_{k}}\right|+\left|v_{j_{t}}\right|\{\mu[A(\alpha)]\}^{-1} \sum_{\substack{j_{k} \in N_{2} \\
k \neq t}}\left|w_{j_{k}}\right|
\end{aligned}
$$




$$
\begin{aligned}
& +\sum_{j_{k} \in N_{2}}\left|a_{j_{s} j_{k}}\right|+\left|v_{j_{s}}\right|\{\mu[A(\alpha)]\}^{-1} \sum_{j_{k} \in N_{2}}\left|w_{j_{k}}\right| \\
& =\left|a_{j_{t} j_{t}}\right|-\sum_{\substack{j_{k} \in N_{2} \\
k \neq t}}\left|a_{j_{t} j_{k}}\right|+\sum_{j_{k} \in N_{2}}\left|a_{j_{s} j_{k}}\right|+\left|v_{j_{t}}\right|\{\mu[A(\alpha)]\}^{-1} \sum_{j_{k} \in N_{2}}\left|w_{j_{k}}\right| \\
& +\left|v_{j_{s}}\right|\{\mu[A(\alpha)]\}^{-1} \sum_{j_{k} \in N_{2}}\left|w_{j_{k}}\right| \\
& =\left|a_{j_{t} j_{t}}\right|-\sum_{\substack{j_{k} \in N_{2} \\
k \neq t}}\left|a_{j_{t} j_{k}}\right|+\sum_{j_{k} \in N_{2}}\left|a_{j_{s} j_{k}}\right|+\left(\left|v_{j_{t}}\right|+\left|v_{j_{s}}\right|\right)\{\mu[A(\alpha)]\}^{-1} \sum_{j_{k} \in N_{2}}\left|w_{j_{k}}\right| \\
& \leq\left|a_{j_{t} j_{t}}\right|-\sum_{\substack{j_{k} \in N_{2} \\
k \neq t}}\left|a_{j_{t} j_{k}}\right|+\sum_{j_{k} \in N_{2}}\left|a_{j_{s} j_{k}}\right|+\left(\left|v_{j_{t}}\right|+\left|v_{j_{s}}\right|\right)\{\mu[A(\alpha)]\}^{-1} \sum_{k \in[m]}\left|w_{j_{k}}\right|\left(\text { by (18) and } N_{2} \subset \alpha^{\prime}\right) \\
& =\left|a_{j_{t} j_{t}}\right|-\sum_{\substack{j_{k} \in N_{2} \\
k \neq t}}\left|a_{j_{t} j_{k}}\right|+\sum_{j_{k} \in N_{2}}\left|a_{j_{s} j_{k}}\right|+\sum_{k \in[l]}\left(\left|a_{j_{t} i_{k}}\right|+\left|a_{j_{s} i_{k}}\right|\right) x_{k} \\
& \leq\left|a_{j_{t} j_{t}}\right|-\sum_{\substack{j_{k} \in N_{2} \\
k \neq t}}\left|a_{j_{t} j_{k}}\right|+\sum_{j_{k} \in N_{2}}\left|a_{j_{s} j_{k}}\right|+\sum_{k \in[l]}\left(\left|a_{j_{t} i_{k}}\right|+\left|a_{j_{s} i_{k}}\right|\right)(\text { by inequality (19)))} \\
& =\left|a_{j_{t} j_{t}}\right|-\beta_{j_{t}}(A)+\beta_{j_{s}}(A)+\sum_{k \in[l]}\left|a_{j_{t} i_{k}}\right|+\sum_{k \in[l]}\left|a_{j_{s} i_{k}}\right| .
\end{aligned}
$$

Similarly,

$$
\begin{aligned}
& \left|a_{s s}^{\prime}\right|-\alpha_{s}(A / \alpha)+\alpha_{t}(A / \alpha) \\
& =\left|a_{s s}^{\prime}\right|-\sum_{\substack{j_{k} \in N_{1}-\alpha \\
k \neq s}}\left|a_{s k}^{\prime}\right|+\sum_{j_{k} \in N_{1}-\alpha}\left|a_{t k}^{\prime}\right| \\
& \leq\left|a_{j_{s} j_{s}}\right|+\left|v_{j_{s}}\right|\{\mu[A(\alpha)]\}^{-1}\left|w_{j_{s}}\right|-\sum_{\substack{j_{k} \in N_{1}-\alpha \\
k \neq s}}\left(\left|a_{j_{s} j_{k}}\right|-\left|v_{j_{s}}\right|\{\mu[A(\alpha)]\}^{-1}\left|w_{j_{k}}\right|\right) \\
& +\sum_{j_{k} \in N_{1}-\alpha}\left(\left|a_{j_{t} j_{k}}\right|+\left|v_{j_{t}}\right|\{\mu[A(\alpha)]\}^{-1}\left|w_{j_{k}}\right|\right) \text { (by inequalities (23) and (24)) } \\
& =\left|a_{j_{s} j_{s}}\right|+\left|v_{j_{s}}\right|\{\mu[A(\alpha)]\}^{-1}\left|w_{j_{s}}\right|-\sum_{\substack{j_{k} \in N_{1}-\alpha \\
k \neq s}}\left|a_{j_{s} j_{k}}\right|+\left|v_{j_{s}}\right|\{\mu[A(\alpha)]\}^{-1} \sum_{\substack{j_{k} \in N_{1}-\alpha \\
k \neq s}}\left|w_{j_{k}}\right| \\
& +\sum_{j_{k} \in N_{1}-\alpha}\left|a_{j_{t} j_{k}}\right|+\left|v_{j_{t}}\right|\{\mu[A(\alpha)]\}^{-1} \sum_{j_{k} \in N_{1}-\alpha}\left|w_{j_{k}}\right| \\
& =\left|a_{j_{s} j_{s}}\right|-\sum_{\substack{j_{k} \in N_{1}-\alpha \\
k \neq s}}\left|a_{j_{s} j_{k}}\right|+\sum_{j_{k} \in N_{1}-\alpha}\left|a_{j j_{k}}\right|+\left|v_{j_{s}}\right|\{\mu[A(\alpha)]\}^{-1} \sum_{j_{k} \in N_{1}-\alpha}\left|w_{j_{k}}\right| \\
& +\left|v_{j_{t}}\right|\{\mu[A(\alpha)]\}^{-1} \sum_{j_{k} \in N_{1}-\alpha}\left|w_{j_{k}}\right| \\
& =\left|a_{j_{s} j_{s}}\right|-\sum_{\substack{j_{k} \in N_{1}-\alpha \\
k \neq s}}\left|a_{j_{s} j_{k}}\right|+\sum_{j_{k} \in N_{1}-\alpha}\left|a_{j_{t} j_{k}}\right|+\left(\left|v_{j_{s}}\right|+\left|v_{j_{t}}\right|\right)\{\mu[A(\alpha)]\}^{-1} \sum_{j_{k} \in N_{1}-\alpha}\left|w_{j_{k}}\right| \\
& \leq\left|a_{j_{s} j_{s}}\right|-\sum_{\substack{j_{k} \in N_{1}-\alpha \\
k \neq s}}\left|a_{j_{s} j_{k}}\right|+\sum_{j_{k} \in N_{1}-\alpha}\left|a_{j_{t} j_{k}}\right|+\left(\left|v_{j_{s}}\right|+\left|v_{j_{t}}\right|\right)\{\mu[A(\alpha)]\}^{-1} \sum_{k \in[m]}\left|w_{j_{k}}\right|\left(\text { by (18) and } N_{1}-\alpha \subset \alpha^{\prime}\right) \\
& =\left|a_{j_{s} j_{s}}\right|-\sum_{\substack{j_{k} \in N_{1}-\alpha \\
k \neq s}}\left|a_{j_{s} j_{k}}\right|+\sum_{j_{k} \in N_{1}-\alpha}\left|a_{j_{t} j_{k}}\right|+\sum_{k \in[l]}\left(\left|a_{j_{s} i_{k}}\right|+\left|a_{j_{t} i_{k}}\right|\right) x_{k} \\
& \leq\left|a_{j_{s} j_{s}}\right|-\sum_{\substack{j_{k} \in N_{1}-\alpha \\
k \neq s}}\left|a_{j_{s} j_{k}}\right|+\sum_{j_{k} \in N_{1}-\alpha}\left|a_{j_{t} j_{k}}\right|+\sum_{k \in[l]}\left(\left|a_{j_{s} i_{k}}\right|+\left|a_{j_{t} i_{k}}\right|\right) \text { (by inequality (19)) } \\
& =\left|a_{j_{s} j_{s}}\right|+\alpha_{j_{t}}(A)-\sum_{\substack{j_{k} \in N_{1}-\alpha \\
k \neq s}}\left|a_{j_{s} j_{k}}\right|+\sum_{k \in[l]}\left|a_{j_{s} i_{k}}\right|
\end{aligned}
$$


Furthermore, by (26)-(29), we have:

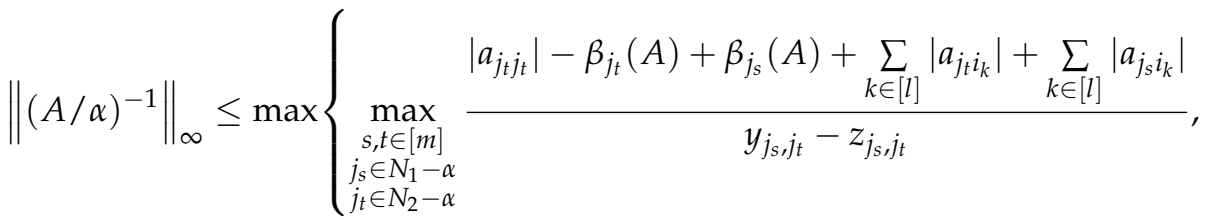

$$
\begin{aligned}
& \begin{array}{l}
\left.\max _{\substack{s, t \in[m] \\
j_{s} \in N_{1}-\alpha \\
j_{t} \in N_{2}-\alpha}} \frac{\left|a_{j_{s} j_{s}}\right|+\alpha_{j_{t}}(A)-\sum_{\substack{j_{k} \in N_{1}-\alpha \\
k \neq s}}\left|a_{j_{s} j_{k}}\right|+\sum_{k \in[l]}\left|a_{j_{s} i_{k}}\right|}{y_{j_{s}, j_{t}}-z_{j_{s}, j_{t}}}\right\} \\
=\max \left\{\max _{\substack{i, j \in \alpha^{\prime} \\
i \in N_{1}-\alpha, j \in N_{2}}} \frac{\left|a_{j j}\right|-\beta_{j}(A)+\beta_{i}(A)+\sum_{k \in \alpha}\left|a_{j k}\right|+\sum_{k \in \alpha}\left|a_{i k}\right|}{y_{i, j}-z_{i . j}},\right.
\end{array} \\
& \left.\max _{\substack{i, j \in \alpha^{\prime} \\
i \in N_{1}-\alpha, j \in N_{2}}} \frac{\left|a_{i i}\right|+\alpha_{j}(A)-\sum_{\substack{k \in N_{1}-\alpha \\
k \neq i}}\left|a_{i k}\right|+\sum_{k \in \alpha}\left|a_{i k}\right|}{y_{i, j}-z_{i, j}}\right\},
\end{aligned}
$$

where

$$
\begin{gathered}
y_{j_{s}, j_{t}}=\left(\left|a_{j_{s}, j_{s}}\right|-\sum_{\substack{j_{k} \in N_{1}-\alpha \\
k \neq s}}\left|a_{j_{s} j_{k}}\right|-\left|v_{j_{s}}\right|\{\mu[A(\alpha)]\}^{-1} \sum_{j_{k} \in N_{1}-\alpha}\left|w_{j_{k}}\right|\right)\left(\left|a_{j_{t} j_{t}}\right|\right. \\
\left.-\sum_{\substack{j_{k} \in N_{2} \\
k \neq t}}\left|a_{j_{t} j_{k}}\right|-\left|v_{j_{t}}\right|\{\mu[A(\alpha)]\}^{-1} \sum_{j_{k} \in N_{2}}\left|w_{j_{k}}\right|\right), \\
z_{j_{s}, j_{t}}=\left(\sum_{j_{k} \in N_{1}-\alpha}\left|a_{j_{t} j_{k}}\right|+\left|v_{j_{t}}\right|\{\mu[A(\alpha)]\}^{-1} \sum_{j_{k} \in N_{1}-\alpha}\left|w_{j_{k}}\right|\right)\left(\sum_{j_{k} \in N_{2}}\left|a_{j_{s} j_{k}}\right|\right. \\
\left.+\left|v_{j_{s}}\right|\{\mu[A(\alpha)]\}^{-1} \sum_{j_{k} \in N_{2}}\left|w_{j_{k}}\right|\right) .
\end{gathered}
$$

By Corollary 1, we have:

$$
\|Q\|_{\infty} \leq 1+\max _{i \in \alpha} \frac{R_{i}\left(A_{12}\right)}{\left|a_{i i}\right|-r_{i}\left(A_{11}\right)} .
$$

Furthermore, by (7), (9), (25), (30), and (31), the conclusion follows.

Theorem 5. Let $A=\left[a_{i j}\right]$ be GDSDD $D_{n}^{N_{1}, N_{2}}$ with $a_{i i} \neq 0, i \in N$, and $\varnothing \neq \alpha \subset N$. Assume that (8) and (19) hold, if $\alpha \subset N_{2}$, then:

$$
\left\|A^{-1}\right\|_{\infty} \leq \gamma(\alpha) \cdot\left(1+\max _{i \in \alpha} \frac{R_{i}\left(A_{12}\right)}{\left|a_{i i}\right|-r_{i}\left(A_{11}\right)}\right) \cdot \delta(\alpha),
$$

where

$$
\delta(\alpha)=\max \left\{\max _{i \in \alpha} \frac{1}{\left|a_{i i}\right|-r_{i}\left(A_{11}\right)}, \xi(\alpha)\right\}
$$




$$
\begin{aligned}
& \xi(\alpha)=\max \left\{\max _{\substack{i, j \in \alpha^{\prime} \\
i \in N_{1}, j \in N_{2}-\alpha}} \frac{\left|a_{j j}\right|+\beta_{i}(A)-\sum_{k \in N_{2}-\alpha, k \neq j}\left|a_{j k}\right|+\sum_{k \in \alpha}\left|a_{j k}\right|}{f_{i, j}-g_{i, j}},\right. \\
& \left.\max _{\substack{i, j \in \alpha^{\prime} \\
i \in N_{1}, j \in N_{2}-\alpha}} \frac{\left|a_{i i}\right|-\alpha_{i}(A)+\alpha_{j}(A)+\sum_{k \in \alpha}\left|a_{i k}\right|+\sum_{k \in \alpha}\left|a_{j k}\right|}{f_{i, j}-g_{i, j}}\right\}, \\
& \begin{array}{c}
f_{i, j}=\left(\left|a_{i i}\right|-\alpha_{i}(A)-\left|v_{i}\right|\{\mu[A(\alpha)]\}^{-1} \sum_{k \in N_{1}}\left|w_{k}\right|\right)\left(\left|a_{j j}\right|-\sum_{\substack{k \in N_{2}-\alpha \\
k \neq j}}\left|a_{j k}\right|-\left|v_{j}\right|\{\mu[A(\alpha)]\}^{-1} \sum_{k \in N_{2}-\alpha}\left|w_{k}\right|\right), \\
g_{i, j}=\left(\beta_{j}(A)+\left|v_{j}\right|\{\mu[A(\alpha)]\}^{-1} \sum_{k \in N_{1}}\left|w_{k}\right|\right)\left(\sum_{k \in N_{2}-\alpha}\left|a_{i k}\right|+\left|v_{i}\right|\{\mu[A(\alpha)]\}^{-1} \sum_{k \in N_{2}-\alpha}\left|w_{k}\right|\right) .
\end{array}
\end{aligned}
$$

Proof. Since $A$ is $G D S D D_{n}^{N_{1}, N_{2}}, \alpha \subset N_{2}$, by Lemmas 5 and 6, we have $A_{11}$ is $S D D$, and $A / \alpha$ is $G D S D D_{|N-\alpha|}^{N_{1}, N_{2}-\alpha}$. Applying the Varah's bound to $A_{11}$, we get:

$$
\left\|A_{11}^{-1}\right\|_{\infty} \leq \max _{i \in \alpha} \frac{1}{\left|a_{i i}\right|-r_{i}\left(A_{11}\right)}
$$

Applying Theorem 3 to $A / \alpha$ yields:

$$
\begin{gathered}
\left\|(A / \alpha)^{-1}\right\|_{\infty} \leq \max \left\{\max _{\substack{s, t \in[m], j_{s} \in N_{1}-\alpha, j_{t} \in N_{2}}} \frac{\left|a_{t t}^{\prime}\right|-\beta_{t}(A / \alpha)+\beta_{s}(A / \alpha)}{\left(\left|a_{s S}^{\prime}\right|-\alpha_{s}(A / \alpha)\right)\left(\left|a_{t t}^{\prime}\right|-\beta_{t}(A / \alpha)\right)-\alpha_{t}(A / \alpha) \beta_{s}(A / \alpha)},\right. \\
\left.\max _{\substack{s, t \in[m], j_{s} \in N_{1}-\alpha, j_{t} \in N_{2}}} \frac{\left|a_{s S}^{\prime}\right|-\alpha_{s}(A / \alpha)+\alpha_{t}(A / \alpha)}{\left(\left|a_{s s}^{\prime}\right|-\alpha_{s}(A / \alpha)\right)\left(\left|a_{t t}^{\prime}\right|-\beta_{t}(A / \alpha)\right)-\alpha_{t}(A / \alpha) \beta_{s}(A / \alpha)}\right\} .
\end{gathered}
$$

By Theorem 2 in [14], we have:

$$
\begin{aligned}
& \left(\left|a_{s s}^{\prime}\right|-\alpha_{s}(A / \alpha)\right)\left(\left|a_{t t}^{\prime}\right|-\beta_{t}(A / \alpha)\right)-\alpha_{t}(A / \alpha) \beta_{s}(A / \alpha) \\
& \geq\left(\left|a_{j_{s} j_{s}}\right|-\sum_{\substack{j_{k} \in N_{1} \\
k \neq s}}\left|a_{j_{s} j_{k}}\right|-\left|v_{j_{s}}\right|\{\mu[A(\alpha)]\}^{-1} \sum_{j_{k} \in N_{1}}\left|w_{j_{k}}\right|\right)\left(\left|a_{j_{t} j_{t}}\right|-\sum_{\substack{j_{k} \in N_{2}-\alpha \\
k \neq t}}\left|a_{j_{t} j_{k}}\right|\right. \\
& \left.-\left|v_{j_{t}}\right|\{\mu[A(\alpha)]\}^{-1} \sum_{\substack{j_{k} \in N_{2}-\alpha\\
}}\left|w_{j_{k}}\right|\right)-\left(\sum_{j_{k} \in N_{1}}\left|a_{j_{t} j_{k}}\right|+\left|v_{j_{t}}\right|\{\mu[A(\alpha)]\}^{-1} \sum_{j_{k} \in N_{1}}\left|w_{j_{k}}\right|\right)\left(\sum_{j_{k} \in N_{2}-\alpha}\left|a_{j_{s} j_{k}}\right|\right. \\
& \left.+\left|v_{j_{s}}\right|\{\mu[A(\alpha)]\}^{-1} \sum_{j_{k} \in N_{2}-\alpha}\left|w_{j_{k}}\right|\right) \\
& >0 .
\end{aligned}
$$

Now, the upper bounds of $\left|a_{t t}^{\prime}\right|-\beta_{t}(A / \alpha)+\beta_{s}(A / \alpha)$ and $\left|a_{s S}^{\prime}\right|-\alpha_{s}(A / \alpha)+\alpha_{t}(A / \alpha)$ are considered.

By (19), (20), (23), and (24), we have:

$$
\begin{aligned}
& \left|a_{t t}^{\prime}\right|-\beta_{t}(A / \alpha)+\beta_{s}(A / \alpha) \\
& =\left|a_{t t}^{\prime}\right|-\sum_{\substack{j_{k} \in N_{2}-\alpha \\
k \neq t}}\left|a_{t k}^{\prime}\right|+\sum_{j_{k} \in N_{2}-\alpha}\left|a_{s k}^{\prime}\right| \\
& \leq\left|a_{j_{t} j_{t}}\right|+\left|v_{j_{t}}\right|\{\mu[A(\alpha)]\}^{-1}\left|w_{j_{t}}\right|-\sum_{\substack{j_{k} \in N_{2}-\alpha \\
k \neq t}}\left(\left|a_{j_{t} j_{k}}\right|-\left|v_{j_{t}}\right|\{\mu[A(\alpha)]\}^{-1}\left|w_{j_{k}}\right|\right)
\end{aligned}
$$


$+\sum_{j_{k} \in N_{2}-\alpha}\left(\left|a_{j_{s} j_{k}}\right|+\left|v_{j_{s}}\right|\{\mu[A(\alpha)]\}^{-1}\left|w_{j_{k}}\right|\right)$ (by inequalities (23) and (24))

$=\left|a_{j_{t} j_{t}}\right|+\left|v_{j_{t}}\right|\{\mu[A(\alpha)]\}^{-1}\left|w_{j_{t}}\right|-\sum_{\substack{j_{k} \in N_{2}-\alpha \\ k \neq t}}\left|a_{j_{t} j_{k}}\right|+\left|v_{j_{t}}\right|\{\mu[A(\alpha)]\}^{-1} \sum_{\substack{j_{k} \in N_{2}-\alpha \\ k \neq t}}\left|w_{j_{k}}\right|$

$+\sum_{j_{k} \in N_{2}-\alpha}\left|a_{j_{s} j_{k}}\right|+\left|v_{j_{s}}\right|\{\mu[A(\alpha)]\}^{-1} \sum_{j_{k} \in N_{2}-\alpha}\left|w_{j_{k}}\right|$

$=\left|a_{j_{t} j_{t}}\right|-\sum_{\substack{j_{k} \in N_{2}-\alpha \\ k \neq t}}\left|a_{j_{t} j_{k}}\right|+\sum_{j_{k} \in N_{2}-\alpha}\left|a_{j_{s} j_{k}}\right|+\left|v_{j_{t}}\right|\{\mu[A(\alpha)]\}^{-1} \sum_{j_{k} \in N_{2}-\alpha}\left|w_{j_{k}}\right|$

$+\left|v_{j_{s}}\right|\{\mu[A(\alpha)]\}^{-1} \sum_{j_{k} \in N_{2}-\alpha}\left|w_{j_{k}}\right|$

$=\left|a_{j_{t} j_{t}}\right|-\sum_{\substack{j_{k} \in N_{2}-\alpha \\ k \neq t}}\left|a_{j_{t} j_{k}}\right|+\sum_{j_{k} \in N_{2}-\alpha}\left|a_{j_{s} j_{k}}\right|+\left(\left|v_{j_{t}}\right|+\left|v_{j_{s}}\right|\right)\{\mu[A(\alpha)]\}^{-1} \sum_{j_{k} \in N_{2}-\alpha}\left|w_{j_{k}}\right|$

$\leq\left|a_{j_{t} j_{t}}\right|-\sum_{j_{k} \in N_{2}-\alpha}\left|a_{j_{t} j_{k}}\right|+\sum_{j_{k} \in N_{2}-\alpha}\left|a_{j_{s} j_{k}}\right|+\left(\left|v_{j_{t}}\right|+\left|v_{j_{s}}\right|\right)\{\mu[A(\alpha)]\}^{-1} \sum_{k \in[m]}\left|w_{j_{k}}\right|\left(\right.$ by (18) and $\left.N_{2}-\alpha \subset \alpha^{\prime}\right)$

$=\left|a_{j_{t} j_{t}}\right|-\sum_{\substack{j_{k} \in N_{2}-\alpha \\ k \neq t}}\left|a_{j_{t} j_{k}}\right|+\sum_{j_{k} \in N_{2}-\alpha}\left|a_{j_{s} j_{k}}\right|+\sum_{k \in[l]}\left(\left|a_{j_{t} i_{k}}\right|+\left|a_{j_{s} i_{k}}\right|\right) x_{k}$

$\leq\left|a_{j_{t} j_{t}}\right|-\sum_{\substack{j_{k} \in N_{2}-\alpha \\ k \neq t}}\left|a_{j_{t} j_{k}}\right|+\sum_{j_{k} \in N_{2}-\alpha}\left|a_{j_{s} j_{k}}\right|+\sum_{k \in[l]}\left(\left|a_{j_{t} i_{k}}\right|+\left|a_{j_{s} i_{k}}\right|\right)$ (by inequlaity (19))

$=\left|a_{j_{t j} j_{t}}\right|+\beta_{j_{s}}(A)-\sum_{\substack{j_{k} \in N_{2}-\alpha \\ k \neq t}}\left|a_{j_{t} j_{k}}\right|+\sum_{k \in[l]}\left|a_{j_{t} i_{k}}\right|$.

Similarly,

$$
\begin{aligned}
& \left|a_{S S}^{\prime}\right|-\alpha_{s}(A / \alpha)+\alpha_{t}(A / \alpha) \\
& =\left|a_{s s}^{\prime}\right|-\sum_{\substack{j_{k} \in N_{1} \\
k \neq s}}\left|a_{s k}^{\prime}\right|+\sum_{j_{k} \in N_{1}}\left|a_{t k}^{\prime}\right| \\
& \leq\left|a_{j_{s} j_{s}}\right|+\left|v_{j_{s}}\right|\{\mu[A(\alpha)]\}^{-1}\left|w_{j_{s}}\right|-\sum_{\substack{j_{k} \in N_{1} \\
k \neq s}}\left(\left|a_{j_{s} j_{k}}\right|-\left|v_{j_{s}}\right|\{\mu[A(\alpha)]\}^{-1}\left|w_{j_{k}}\right|\right) \\
& +\sum_{j_{k} \in N_{1}}\left(\left|a_{j_{t} j_{k}}\right|+\left|v_{j_{t}}\right|\{\mu[A(\alpha)]\}^{-1}\left|w_{j_{k}}\right|\right) \text { (by inequalities (23) and (24)) } \\
& =\left|a_{j_{s} j_{s}}\right|+\left|v_{j_{s}}\right|\{\mu[A(\alpha)]\}^{-1}\left|w_{j_{s}}\right|-\sum_{\substack{j_{k} \in N_{1} \\
k \neq s}}\left|a_{j_{s} j_{k}}\right|+\left|v_{j_{s}}\right|\{\mu[A(\alpha)]\}^{-1} \sum_{\substack{j_{k} \in N_{1} \\
k \neq s}}\left|w_{j_{k}}\right| \\
& +\sum_{j_{k} \in N_{1}}\left|a_{j_{t} j_{k}}\right|+\left|v_{j_{t}}\right|\{\mu[A(\alpha)]\}^{-1} \sum_{j_{k} \in N_{1}}\left|w_{j_{k}}\right| \\
& =\left|a_{j_{s} j_{s}}\right|-\sum_{\substack{j_{k} \in N_{1} \\
k \neq s}}\left|a_{j_{s} j_{k}}\right|+\sum_{j_{k} \in N_{1}}\left|a_{j_{t} j_{k}}\right|+\left|v_{j_{s}}\right|\{\mu[A(\alpha)]\}^{-1} \sum_{j_{k} \in N_{1}}\left|w_{j_{k}}\right| \\
& +\left|v_{j_{t}}\right|\{\mu[A(\alpha)]\}^{-1} \sum_{j_{k} \in N_{1}}\left|w_{j_{k}}\right| \\
& =\left|a_{j_{s} j_{s}}\right|-\sum_{\substack{j_{k} \in N_{1} \\
k \neq s}}\left|a_{j_{s} j_{k}}\right|+\sum_{j_{k} \in N_{1}}\left|a_{j_{t} j_{k}}\right|+\left(\left|v_{j_{s}}\right|+\left|v_{j_{t}}\right|\right)\{\mu[A(\alpha)]\}^{-1} \sum_{j_{k} \in N_{1}}\left|w_{j_{k}}\right| \\
& \leq\left|a_{j_{s} j_{s}}\right|-\sum_{\substack{j_{k} \in N_{1} \\
k \neq s}}\left|a_{j_{s} j_{k}}\right|+\sum_{j_{k} \in N_{1}}\left|a_{j_{t} j_{k}}\right|+\left(\left|v_{j_{s}}\right|+\left|v_{j_{t}}\right|\right)\{\mu[A(\alpha)]\}^{-1} \sum_{k \in[m]}\left|w_{j_{k}}\right|\left(\text { by (18) and } N_{1} \subset \alpha^{\prime}\right)
\end{aligned}
$$




$$
\begin{aligned}
& =\left|a_{j_{s} j_{s}}\right|-\sum_{\substack{j_{k} \in N_{1} \\
k \neq s}}\left|a_{j_{s} j_{k}}\right|+\sum_{j_{k} \in N_{1}}\left|a_{j_{t} j_{k}}\right|+\sum_{k \in[l]}\left(\left|a_{j_{s} i_{k}}\right|+\left|a_{j_{t} i_{k}}\right|\right) x_{k} \\
& \leq\left|a_{j_{s} j_{s}}\right|-\sum_{\substack{j_{k} \in N_{1} \\
k \neq s}}\left|a_{j_{s} j_{k}}\right|+\sum_{j_{k} \in N_{1}}\left|a_{j_{t} j_{k}}\right|+\sum_{k \in[l]}\left(\left|a_{j_{s} i_{k}}\right|+\left|a_{j_{t} i_{k}}\right|\right) \text { (by inequalities (19)) } \\
& =\left|a_{j_{s} j_{s}}\right|-\alpha_{j_{s}}(A)+\alpha_{j_{t}}(A)+\sum_{k \in[l]}\left|a_{j_{s} i_{k}}\right|+\sum_{k \in[l]}\left|a_{j t i_{k}}\right| .
\end{aligned}
$$

Furthermore, by (33)-(36), we have:

$$
\begin{aligned}
& \left\|(A / \alpha)^{-1}\right\|_{\infty} \leq \max \left\{\max _{\substack{s, t \in[m] \\
j_{s} \in N_{1}, j_{t} \in N_{2}-\alpha}} \frac{\left|a_{j_{t} j_{t}}\right|+\beta_{j_{s}}(A)-\sum_{\substack{j_{k} \in N_{2}-\alpha \\
k \neq t}}\left|a_{j_{t} j_{k}}\right|+\sum_{k \in[l]}\left|a_{j_{t} i_{k}}\right|}{f_{j_{s}, j_{t}}-g_{j_{s}, j_{t}}},\right. \\
& \left.\max _{\substack{s, t \in[m] \\
j_{s} \in N_{1}, j_{t} \in N_{2}-\alpha}} \frac{\left|a_{j_{s} j_{s}}\right|-\alpha_{j_{s}}(A)+\alpha_{j_{t}}(A)+\sum_{k \in[l]}\left|a_{j_{s} i_{k}}\right|+\sum_{k \in[l]}\left|a_{j_{t} i_{k}}\right|}{f_{j_{s}, j_{t}}-g_{j_{s}, j_{t}}}\right\} \\
& =\max \left\{\max _{\substack{i, j \in \alpha^{\prime} \\
i \in N_{1}, j \in N_{2}-\alpha}} \frac{\left|a_{j j}\right|+\beta_{i}(A)-\sum_{\substack{k \in N_{2}-\alpha \\
k \neq j}}\left|a_{j k}\right|+\sum_{k \in \alpha}\left|a_{j k}\right|}{f_{i, j}-g_{i, j}},\right. \\
& \left.\max _{\substack{i, j \in \alpha^{\prime} \\
i \in N_{1}, j \in N_{2}-\alpha}} \frac{\left|a_{i i}\right|-\alpha_{i}(A)+\alpha_{j}(A)+\sum_{k \in \alpha}\left|a_{i k}\right|+\sum_{k \in \alpha}\left|a_{j k}\right|}{f_{i, j}-g_{i, j}}\right\},
\end{aligned}
$$

where

$$
\begin{gathered}
f_{j_{s}, j_{t}}=\left(\left|a_{j_{s} j_{s}}\right|-\sum_{\substack{j_{k} \in N_{1} \\
k \neq s}}\left|a_{j_{s} j_{k}}\right|-\left|v_{j_{s}}\right|\{\mu[A(\alpha)]\}^{-1} \sum_{j_{k} \in N_{1}}\left|w_{j_{k}}\right|\right)\left(\left|a_{j_{t} j_{t}}\right|\right. \\
\left.-\sum_{\substack{j_{k} \in N_{2}-\alpha \\
k \neq t}}\left|a_{j_{t} j_{k}}\right|-\left|v_{j_{t}}\right|\{\mu[A(\alpha)]\}^{-1} \sum_{j_{k} \in N_{2}-\alpha}\left|w_{j_{k}}\right|\right), \\
g_{j_{s}, j_{t}}=\left(\sum_{j_{k} \in N_{1}}\left|a_{j_{t} j_{k}}\right|+\left|v_{j_{t}}\right|\{\mu[A(\alpha)]\}^{-1} \sum_{j_{k} \in N_{1}}\left|w_{j_{k}}\right|\right)\left(\sum_{j_{k} \in N_{2}-\alpha}\left|a_{j_{s} j_{k}}\right|\right. \\
\left.+\left|v_{j_{s}}\right|\{\mu[A(\alpha)]\}^{-1} \sum_{j_{k} \in N_{2}-\alpha}\left|w_{j_{k}}\right|\right) .
\end{gathered}
$$

By Corollary 1, we have:

$$
\|Q\|_{\infty} \leq 1+\max _{i \in \alpha} \frac{R_{i}\left(A_{12}\right)}{\left|a_{i i}\right|-r_{i}\left(A_{11}\right)} .
$$

Furthermore, by (7), (9), (32), (37), and (38), the conclusion follows.

Theorem 6. Let $A=\left[a_{i j}\right]$ be GDSDD $D_{n}^{N_{1}, N_{2}}$ with $a_{i i} \neq 0, i \in N$, and $\varnothing \neq \alpha \subset N$. Assume that (8) and (19) hold, if $\alpha \nsubseteq N_{1}, \alpha \nsubseteq N_{2}, N_{1} \nsubseteq \subset$, and $N_{2} \nsubseteq \propto \alpha$, then:

$$
\left\|A^{-1}\right\|_{\infty} \leq \gamma(\alpha) \cdot(1+\varphi(\alpha)) \cdot \zeta(\alpha),
$$

where 


$$
\begin{aligned}
& \varphi(\alpha)=\max \left\{\max _{\substack{i \in N_{1} \cap \alpha \\
j \in N_{2} \cap \alpha}} \frac{\left(\left|a_{j j}\right|-\beta_{j}\right) R_{i}\left(A_{12}\right)+\beta_{i} R_{j}\left(A_{12}\right)}{\left(\left|a_{i i}\right|-\alpha_{i}\right)\left(\left|a_{j j}\right|-\beta_{j}\right)-\beta_{i} \alpha_{j}} \max _{\substack{i \in N_{1} \cap \alpha \\
j \in N_{2} \cap \alpha}} \frac{\left(\left|a_{i i}\right|-\alpha_{i}\right) R_{j}\left(A_{12}\right)+\alpha_{j} R_{i}\left(A_{12}\right)}{\left(\left|a_{i i}\right|-\alpha_{i}\right)\left(\left|a_{j j}\right|-\beta_{j}\right)-\beta_{i} \alpha_{j}}\right\}, \\
& \zeta(\alpha)=\max \{\omega(\alpha), \tau(\alpha)\} \\
& \omega(\alpha)=\max \left\{\max _{\substack{i \in N_{1} \cap \alpha \\
j \in N_{2} \cap \alpha}} \frac{\left|a_{j j}\right|-\beta_{j}+\beta_{i}}{\left(\left|a_{i i}\right|-\alpha_{i}\right)\left(\left|a_{j j}\right|-\beta_{j}\right)-\beta_{i} \alpha_{j}}, \max _{\substack{i \in N_{1} \cap \alpha \\
j \in N_{2} \cap \alpha}} \frac{\left|a_{i i}\right|-\alpha_{i}+\alpha_{j}}{\left(\left|a_{i i}\right|-\alpha_{i}\right)\left(\left|a_{j j}\right|-\beta_{j}\right)-\beta_{i} \alpha_{j}}\right\}, \\
& \tau(\alpha)=\max \left\{\max _{\substack{i, j \in \alpha^{\prime} \\
i \in N_{1}-\alpha, j \in N_{2}-\alpha}} \frac{\left|a_{j j}\right|+\beta_{i}(A)-\sum_{k \in N_{2}-\alpha}\left|a_{j k}\right|+\sum_{k \in N_{1} \cap \alpha}\left|a_{i k}\right|+\sum_{k \in \alpha}\left|a_{j k}\right|}{p_{i, j}-q_{i, j}},\right. \\
& \left.\max _{\substack{i, j \in \alpha^{\prime} \\
i \in N_{1}-\alpha, j \in N_{2}-\alpha}} \frac{\left|a_{i i}\right|+\alpha_{j}(A)-\sum_{k \in N_{2}-\alpha}\left|a_{i k}\right|+\sum_{k \in N_{2} \cap \alpha}\left|a_{j k}\right|+\sum_{k \in \alpha}\left|a_{i k}\right|}{p_{i, j}-q_{i, j}}\right\}, \\
& p_{i, j}=\left(\left|a_{i i}\right|-\sum_{\substack{k \in N_{1}-\alpha \\
k \neq s}}\left|a_{i k}\right|-\left|v_{i}\right|\{\mu[A(\alpha)]\}^{-1} \sum_{k \in N_{1}-\alpha}\left|w_{k}\right|\right)\left(\left|a_{j j}\right|-\sum_{\substack{k \in N_{2}-\alpha \\
k \neq t}}\left|a_{j k}\right|-\left|v_{j}\right|\{\mu[A(\alpha)]\}^{-1} \sum_{k \in N_{2}-\alpha}\left|w_{k}\right|\right), \\
& q_{i, j}=\left(\sum_{k \in N_{1}-\alpha}\left|a_{j k}\right|+\left|v_{j}\right|\{\mu[A(\alpha)]\}^{-1} \sum_{k \in N_{1}-\alpha}\left|w_{k}\right|\right)\left(\sum_{k \in N_{2}-\alpha}\left|a_{i k}\right|+\left|v_{i}\right|\{\mu[A(\alpha)]\}^{-1} \sum_{k \in N_{2}-\alpha}\left|w_{k}\right|\right) .
\end{aligned}
$$

Proof. Since $A$ is GDSDD $D_{n}^{N_{1}, N_{2}}, \alpha \nsubseteq N_{1}, \alpha \nsubseteq N_{2}, N_{1} \nsubseteq \subset \alpha$ and $N_{2} \nsubseteq \propto \alpha$, by Lemmas 5 and 6, we have $A_{11}$ is $G D S D D_{|\alpha|}^{N_{1} \cap \alpha, N_{2} \cap \alpha}$, and $A / \alpha$ is $G D S D D_{|N-\alpha|}^{N_{1}-\alpha, N_{2}-\alpha}$. Applying Theorem 3 to $A_{11}$, we get:

$$
\left\|A_{11}^{-1}\right\|_{\infty} \leq \max \left\{\max _{\substack{i \in N_{1} \cap \alpha \\ j \in N_{2} \cap \alpha}} \frac{\left|a_{j j}\right|-\beta_{j}+\beta_{i}}{\left(\left|a_{i i}\right|-\alpha_{i}\right)\left(\left|a_{j j}\right|-\beta_{j}\right)-\beta_{i} \alpha_{j}} \max _{\substack{i \in N_{1} \cap \alpha \\ j \in N_{2} \cap \alpha}} \frac{\left|a_{i i}\right|-\alpha_{i}+\alpha_{j}}{\left(\left|a_{i i}\right|-\alpha_{i}\right)\left(\left|a_{j j}\right|-\beta_{j}\right)-\beta_{i} \alpha_{j}}\right\} .
$$

Applying Theorem 3 to $A / \alpha$ yields:

$$
\begin{gathered}
\left\|(A / \alpha)^{-1}\right\|_{\infty} \leq \max \left\{\max _{\substack{s, t \in[m] \\
j_{s} \in N_{1}-\alpha, j_{t} \in N_{2}-\alpha}} \frac{\left|a_{t t}^{\prime}\right|-\beta_{t}(A / \alpha)+\beta_{s}(A / \alpha)}{\left(\left|a_{s S}^{\prime}\right|-\alpha_{s}(A / \alpha)\right)\left(\left|a_{t t}^{\prime}\right|-\beta_{t}(A / \alpha)\right)-\alpha_{t}(A / \alpha) \beta_{s}(A / \alpha)^{\prime}}\right. \\
\left.\max _{\substack{s, t \in[m] \\
j_{s} \in N_{1}-\alpha, j_{t} \in N_{2}-\alpha}} \frac{\left|a_{S S}^{\prime}\right|-\alpha_{s}(A / \alpha)+\alpha_{t}(A / \alpha)}{\left(\left|a_{S S}^{\prime}\right|-\alpha_{s}(A / \alpha)\right)\left(\left|a_{t t}^{\prime}\right|-\beta_{t}(A / \alpha)\right)-\alpha_{t}(A / \alpha) \beta_{s}(A / \alpha)}\right\} .
\end{gathered}
$$

By Theorem 2 in [14], we have: 


$$
\begin{aligned}
& \left(\left|a_{S S}^{\prime}\right|-\alpha_{s}(A / \alpha)\right)\left(\left|a_{t t}^{\prime}\right|-\beta_{t}(A / \alpha)\right)-\alpha_{t}(A / \alpha) \beta_{s}(A / \alpha) \\
& \geq\left(\left|a_{j_{s} j_{s}}\right|-\sum_{\substack{j_{k} \in N_{1}-\alpha \\
k \neq s}}\left|a_{j_{s} j_{k}}\right|-\left|v_{j_{s}}\right|\{\mu[A(\alpha)]\}^{-1} \sum_{j_{k} \in N_{1}-\alpha}\left|w_{j_{k}}\right|\right)\left(\left|a_{j_{t} j_{t}}\right|-\sum_{\substack{j_{k} \in N_{2}-\alpha \\
k \neq t}}\left|a_{j_{t} j_{k}}\right|\right. \\
& \left.-\left|v_{j_{t}}\right|\{\mu[A(\alpha)]\}^{-1} \sum_{j_{k} \in N_{2}-\alpha}\left|w_{j_{k}}\right|\right)-\left(\sum_{j_{k} \in N_{1}-\alpha}\left|a_{j_{t} j_{k}}\right|+\left|v_{j_{t}}\right|\{\mu[A(\alpha)]\}^{-1} \sum_{j_{k} \in N_{1}-\alpha}\left|w_{j_{k}}\right|\right)\left(\sum_{j_{k} \in N_{2}-\alpha}\left|a_{j_{s} j_{k}}\right|\right. \\
& \left.+\left|v_{j_{s}}\right|\{\mu[A(\alpha)]\}^{-1} \sum_{j_{k} \in N_{2}-\alpha}\left|w_{j_{k}}\right|\right) \\
& >0 \text {. }
\end{aligned}
$$

Now, the upper bounds of $\left|a_{t t}^{\prime}\right|-\beta_{t}(A / \alpha)+\beta_{S}(A / \alpha)$ and $\left|a_{S S}^{\prime}\right|-\alpha_{S}(A / \alpha)+\alpha_{t}(A / \alpha)$ are considered.

By (19), (20), (23), and (24), we have:

$\left|a_{t t}^{\prime}\right|-\beta_{t}(A / \alpha)+\beta_{s}(A / \alpha)$

$=\left|a_{t t}^{\prime}\right|-\sum_{\substack{j_{k} \in N_{2}-\alpha \\ k \neq t}}\left|a_{t k}^{\prime}\right|+\sum_{j_{k} \in N_{2}-\alpha}\left|a_{s k}^{\prime}\right|$

$\leq\left|a_{j_{t} j_{t}}\right|+\left|v_{j_{t}}\right|\{\mu[A(\alpha)]\}^{-1}\left|w_{j_{t}}\right|-\sum_{\substack{j_{k} \in N_{2}-\alpha \\ k \neq t}}\left(\left|a_{j_{t} j_{k}}\right|-\left|v_{j_{t}}\right|\{\mu[A(\alpha)]\}^{-1}\left|w_{j_{k}}\right|\right)$

$+\sum_{j_{k} \in N_{2}-\alpha}\left(\left|a_{j_{s} j_{k}}\right|+\left|v_{j_{s}}\right|\{\mu[A(\alpha)]\}^{-1}\left|w_{j_{k}}\right|\right)$ (by inequalities (23) and (24))

$=\left|a_{j_{t} j_{t}}\right|+\left|v_{j_{t}}\right|\{\mu[A(\alpha)]\}^{-1}\left|w_{j_{t}}\right|-\sum_{\substack{j_{k} \in N_{2}-\alpha \\ k \neq t}}\left|a_{j_{t} j_{k}}\right|+\left|v_{j_{t}}\right|\{\mu[A(\alpha)]\}^{-1} \sum_{\substack{j_{k} \in N_{2}-\alpha \\ k \neq t}}\left|w_{j_{k}}\right|$

$+\sum_{j_{k} \in N_{2}-\alpha}\left|a_{j_{s} j_{k}}\right|+\left|v_{j_{s}}\right|\{\mu[A(\alpha)]\}^{-1} \sum_{j_{k} \in N_{2}-\alpha}\left|w_{j_{k}}\right|$

$=\left|a_{j_{t} j_{t}}\right|-\sum_{\substack{j_{k} \in N_{2}-\alpha \\ k \neq t}}\left|a_{j_{t} j_{k}}\right|+\sum_{j_{k} \in N_{2}-\alpha}\left|a_{j_{s} j_{k}}\right|+\left|v_{j_{t}}\right|\{\mu[A(\alpha)]\}^{-1} \sum_{j_{k} \in N_{2}-\alpha}\left|w_{j_{k}}\right|$

$+\left|v_{j_{s}}\right|\{\mu[A(\alpha)]\}^{-1} \sum_{j_{k} \in N_{2}-\alpha}\left|w_{j_{k}}\right|$

$=\left|a_{j_{t} j_{t}}\right|-\sum_{\substack{j_{k} \in N_{2}-\alpha \\ k \neq t}}\left|a_{j_{t} j_{k}}\right|+\sum_{j_{k} \in N_{2}-\alpha}\left|a_{j_{s} j_{k}}\right|+\left(\left|v_{j_{t}}\right|+\left|v_{j_{s}}\right|\right)\{\mu[A(\alpha)]\}^{-1} \sum_{j_{k} \in N_{2}-\alpha}\left|w_{j_{k}}\right|$

$\leq\left|a_{j_{t} j_{t}}\right|-\sum_{j_{k} \in N_{2}-\alpha}\left|a_{j_{t} j_{k}}\right|+\sum_{j_{k} \in N_{2}-\alpha}\left|a_{j_{s} j_{k}}\right|+\left(\left|v_{j_{t}}\right|+\left|v_{j_{s}}\right|\right)\{\mu[A(\alpha)]\}^{-1} \sum_{k \in[m]}\left|w_{j_{k}}\right|\left(\right.$ by (18) and $\left.N_{2}-\alpha \subset \alpha^{\prime}\right)$

$=\left|a_{j_{t} j_{t}}\right|-\sum_{\substack{j_{k} \in N_{2}-\alpha \\ k \neq t}}\left|a_{j_{t} j_{k}}\right|+\sum_{j_{k} \in N_{2}-\alpha}\left|a_{j_{s} j_{k}}\right|+\sum_{k \in[l]}\left(\left|a_{j_{t} i_{k}}\right|+\left|a_{j_{s} i_{k}}\right|\right) x_{k}$

$\leq\left|a_{j_{t} j_{t}}\right|-\sum_{j_{k} \in N_{2}-\alpha}\left|a_{j_{t} j_{k}}\right|+\sum_{j_{k} \in N_{2}-\alpha}\left|a_{j_{s} j_{k}}\right|+\sum_{k \in[l]}\left(\left|a_{j_{t} i_{k}}\right|+\left|a_{j_{s} i_{k}}\right|\right)$ (by inequality (19))

$=\left|a_{j_{t} j_{t}}\right|-\sum_{\substack{j_{k} \in N_{2}-\alpha \\ k \neq t}}\left|a_{j_{t} j_{k}}\right|+\sum_{j_{k} \in N_{2}-\alpha}\left|a_{j_{s} j_{k}}\right|+\sum_{k \in[l]}\left|a_{j_{t} i_{k}}\right|+\sum_{k \in[l]}\left|a_{j_{s} i_{k}}\right|$.

Similarly, 


$$
\begin{aligned}
& \left|a_{s s}^{\prime}\right|-\alpha_{s}(A / \alpha)+\alpha_{t}(A / \alpha) \\
& =\left|a_{s s}^{\prime}\right|-\sum_{\substack{j_{k} \in N_{1}-\alpha \\
k \neq s}}\left|a_{s k}^{\prime}\right|+\sum_{j_{k} \in N_{1}-\alpha}\left|a_{t k}^{\prime}\right| \\
& \leq\left|a_{j_{s} j_{s}}\right|+\left|v_{j_{s}}\right|\{\mu[A(\alpha)]\}^{-1}\left|w_{j_{s}}\right|-\sum_{\substack{j_{k} \in N_{1}-\alpha \\
k \neq s}}\left(\left|a_{j_{s} j_{k}}\right|-\left|v_{j_{s}}\right|\{\mu[A(\alpha)]\}^{-1}\left|w_{j_{k}}\right|\right) \\
& +\sum_{j_{k} \in N_{1}-\alpha}\left(\left|a_{j_{t} j_{k}}\right|+\left|v_{j_{t}}\right|\{\mu[A(\alpha)]\}^{-1}\left|w_{j_{k}}\right|\right) \text { (by inequalities (23) and (24)) } \\
& =\left|a_{j_{s} j_{s}}\right|+\left|v_{j_{s}}\right|\{\mu[A(\alpha)]\}^{-1}\left|w_{j_{s}}\right|-\sum_{\substack{j_{k} \in N_{1}-\alpha \\
k \neq s}}\left|a_{j_{s} j_{k}}\right|+\left|v_{j_{s}}\right|\{\mu[A(\alpha)]\}^{-1} \sum_{\substack{j_{k} \in N_{1}-\alpha \\
k \neq s}}\left|w_{j_{k}}\right| \\
& +\sum_{j_{k} \in N_{1}-\alpha}\left|a_{j_{t} j_{k}}\right|+\left|v_{j_{t}}\right|\{\mu[A(\alpha)]\}^{-1} \sum_{j_{k} \in N_{1}-\alpha}\left|w_{j_{k}}\right| \\
& =\left|a_{j_{s} j_{s}}\right|-\sum_{\substack{j_{k} \in N_{1}-\alpha \\
k \neq s}}\left|a_{j_{s} j_{k}}\right|+\sum_{j_{k} \in N_{1}-\alpha}\left|a_{j_{t} j_{k}}\right|+\left|v_{j_{s}}\right|\{\mu[A(\alpha)]\}^{-1} \sum_{j_{k} \in N_{1}-\alpha}\left|w_{j_{k}}\right| \\
& +\left|v_{j_{t}}\right|\{\mu[A(\alpha)]\}^{-1} \sum_{j_{k} \in N_{1}-\alpha}\left|w_{j_{k}}\right| \\
& =\left|a_{j_{s} j_{s}}\right|-\sum_{\substack{j_{k} \in N_{1}-\alpha \\
k \neq s}}\left|a_{j_{s} j_{k}}\right|+\sum_{j_{k} \in N_{1}-\alpha}\left|a_{j_{t} j_{k}}\right|+\left(\left|v_{j_{s}}\right|+\left|v_{j_{t}}\right|\right)\{\mu[A(\alpha)]\}^{-1} \sum_{j_{k} \in N_{1}-\alpha}\left|w_{j_{k}}\right| \\
& \leq\left|a_{j_{s} j_{s}}\right|-\sum_{\substack{j_{k} \in N_{1}-\alpha \\
k \neq s}}\left|a_{j_{s} j_{k}}\right|+\sum_{j_{k} \in N_{1}-\alpha}\left|a_{j_{t} j_{k}}\right|+\left(\left|v_{j_{s}}\right|+\left|v_{j_{t}}\right|\right)\{\mu[A(\alpha)]\}^{-1} \sum_{k \in[m]}\left|w_{j_{k}}\right|\left(\text { by (18) and } N_{1}-\alpha \subset \alpha^{\prime}\right) \\
& =\left|a_{j_{s} j_{s}}\right|-\sum_{\substack{j_{k} \in N_{1}-\alpha \\
k \neq s}}\left|a_{j_{s} j_{k}}\right|+\sum_{j_{k} \in N_{1}-\alpha}\left|a_{j_{t} j_{k}}\right|+\sum_{k \in[l]}\left(\left|a_{j_{s} i_{k}}\right|+\left|a_{j_{t} i_{k}}\right|\right) x_{k} \\
& \leq\left|a_{j_{s} j_{s}}\right|-\sum_{\substack{j_{k} \in N_{1}-\alpha \\
k \neq s}}\left|a_{j_{s} j_{k}}\right|+\sum_{j_{k} \in N_{1}-\alpha}\left|a_{j_{t} j_{k}}\right|+\sum_{k \in[l]}\left(\left|a_{j_{s} i_{k}}\right|+\left|a_{j_{t} i_{k}}\right|\right) \text { (by inquality (19)) } \\
& =\left|a_{j_{s} j_{s}}\right|-\sum_{\substack{j_{k} \in N_{1}-\alpha \\
k \neq s}}\left|a_{j_{s} j_{k}}\right|+\sum_{j_{k} \in N_{1}-\alpha}\left|a_{j j_{j}}\right|+\sum_{k \in[l]}\left|a_{j_{s} i_{k}}\right|+\sum_{k \in[l]}\left|a_{j i_{i}}\right| . \\
& \text { Furthermore, by (40)-(43), we have: }
\end{aligned}
$$

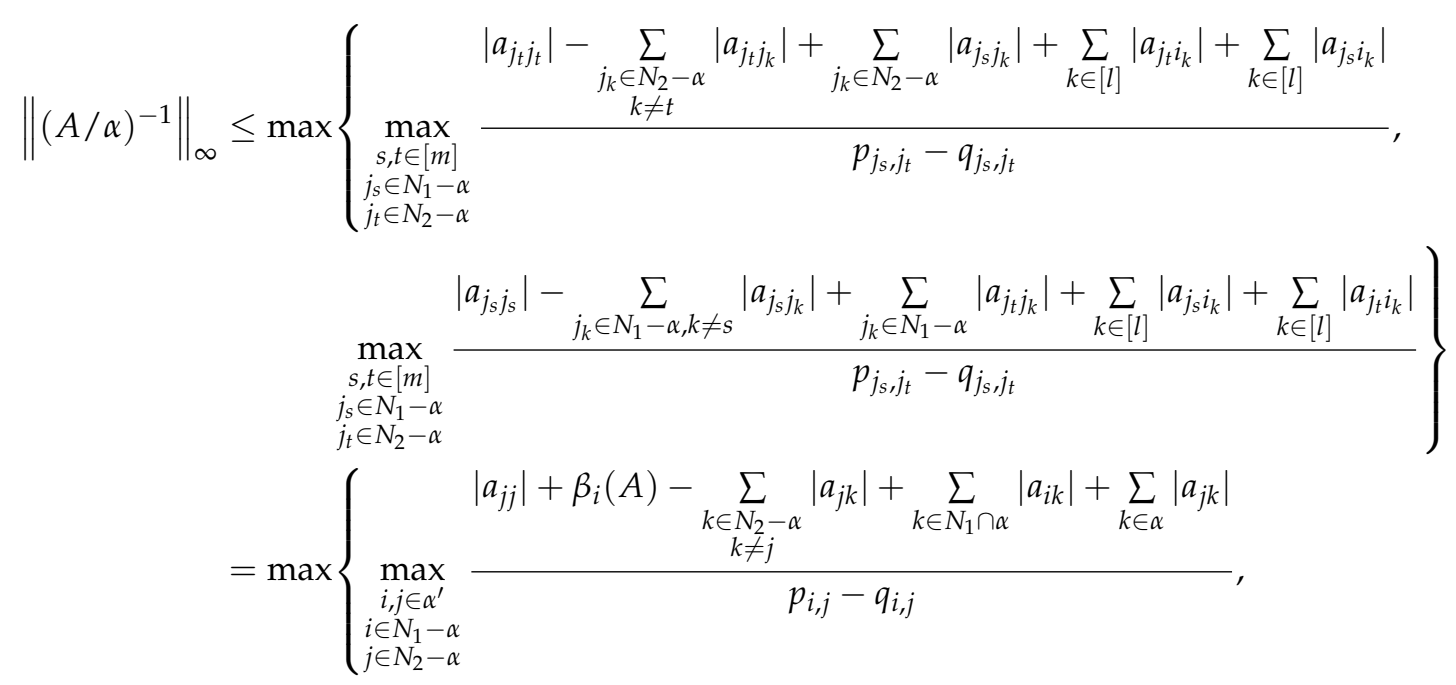




$$
\left.\max _{\substack{i, j \in \alpha^{\prime} \\ i \in N_{1}-\alpha \\ j \in N_{2}-\alpha}} \frac{\left|a_{i i}\right|-\sum_{k \in N_{2}-\alpha}\left|a_{i k}\right|+\alpha_{j}(A)+\sum_{k \in N_{2} \cap \alpha}\left|a_{j k}\right|+\sum_{k \in \alpha}\left|a_{i k}\right|}{p_{i, j}-q_{i, j}}\right\},
$$

where

$$
\begin{gathered}
p_{j_{s}, j_{t}}=\left(\left|a_{j_{s} j_{s}}\right|-\sum_{\substack{j_{k} \in N_{1}-\alpha \\
k \neq s}}\left|a_{j_{s} j_{k}}\right|-\left|v_{j_{s}}\right|\{\mu[A(\alpha)]\}^{-1} \sum_{j_{k} \in N_{1}-\alpha}\left|w_{j_{k}}\right|\right)\left(\left|a_{j_{j} j_{t}}\right|\right. \\
\left.-\sum_{\substack{j_{k} \in N_{2}-\alpha \\
k \neq t}}\left|a_{j_{t} j_{k}}\right|-\left|v_{j_{t}}\right|\{\mu[A(\alpha)]\}^{-1} \sum_{j_{k} \in N_{2}-\alpha}\left|w_{j_{k}}\right|\right), \\
q_{j_{s}, j_{t}}=\left(\sum_{j_{k} \in N_{1}-\alpha}\left|a_{j_{t} j_{k}}\right|+\left|v_{j_{t}}\right|\{\mu[A(\alpha)]\}^{-1} \sum_{j_{k} \in N_{1}-\alpha}\left|w_{j_{k}}\right|\right)\left(\sum_{j_{k} \in N_{2}-\alpha}\left|a_{j_{s} j_{k}}\right|\right. \\
\left.+\left|v_{j_{s}}\right|\{\mu[A(\alpha)]\}^{-1} \sum_{j_{k} \in N_{2}-\alpha}\left|w_{j_{k}}\right|\right) .
\end{gathered}
$$

By Corollary 1, we have:

$$
\|Q\|_{\infty} \leq \max \left\{\max _{\substack{i \in N_{1} \cap \alpha \\ j \in N_{2} \cap \alpha}} \frac{\left(\left|a_{j j}\right|-\beta_{j}\right) R_{i}\left(A_{12}\right)+\beta_{i} R_{j}\left(A_{12}\right)}{\left(\left|a_{i i}\right|-\alpha_{i}\right)\left(\left|a_{j j}\right|-\beta_{j}\right)-\beta_{i} \alpha_{j}} \max _{\substack{i \in N_{1} \cap \alpha \\ j \in N_{2} \cap \alpha}} \frac{\left(\left|a_{i i}\right|-\alpha_{i}\right) R_{j}\left(A_{12}\right)+\alpha_{j} R_{i}\left(A_{12}\right)}{\left(\left|a_{i i}\right|-\alpha_{i}\right)\left(\left|a_{j j}\right|-\beta_{j}\right)-\beta_{i} \alpha_{j}}\right\} \text {. }
$$

Furthermore, by (7), (9), (39), (44), and (45), the conclusion follows.

Theorem 7. Let $A=\left[a_{i j}\right]$ be GDSDD $D_{n}^{N_{1}, N_{2}}$ with $a_{i i} \neq 0, i \in N$, and $\varnothing \neq \alpha \subset N$. Assume that (8) holds, if $N_{1} \subset \alpha$ or $N_{2} \subset \alpha$, then:

$$
\left\|A^{-1}\right\|_{\infty} \leq \gamma(\alpha) \cdot(1+\varphi(\alpha)) \cdot \max \left\{\phi(\alpha), \max _{j \in \alpha^{\prime}} \frac{1}{\left|a_{j j}\right|-\sum_{\substack{k \in \alpha^{\prime} \\ k \neq j}}\left|a_{j k}\right|-w_{j}(A)}\right\},
$$

where

$$
\begin{gathered}
\phi(\alpha)=\max \left\{\max _{\substack{i \in N_{1} \cap \alpha \alpha \\
j \in N_{2} \cap \alpha}} \frac{\left|a_{j j}\right|-\beta_{j}+\beta_{i}}{\left(\left|a_{i i}\right|-\alpha_{i}\right)\left(\left|a_{j j}\right|-\beta_{j}\right)-\beta_{i} \alpha_{j}}, \max _{\substack{i \in N_{1} \cap \alpha \\
j \in N_{2} \cap \alpha}} \frac{\left|a_{i i}\right|-\alpha_{i}+\alpha_{j}}{\left(\left|a_{i i}\right|-\alpha_{i}\right)\left(\left|a_{j j}\right|-\beta_{j}\right)-\beta_{i} \alpha_{j}}\right\}, \\
w_{j}(A)=\max _{i \in \alpha} \frac{\sum_{\substack{k \in \alpha^{\prime} \\
\left|a_{i i}\right|-a_{i k} \mid}}\left|a_{i k}\right|}{\sum_{\substack{k \in \alpha \\
k \neq i}}\left|a_{j k}\right| .}
\end{gathered}
$$

Proof. Since $A$ is $G D S D D_{n}^{N_{1}, N_{2}}, N_{1} \subset \alpha$ or $N_{2} \subset \alpha$, by Lemmas 5 and 6 , we have $A_{11}$ is $G D S D D_{|\alpha|}^{N_{1} \cap \alpha, N_{2} \cap \alpha}$ and $A / \alpha$ is $S D D$, consequently, nonsingular. Let:

$$
A / \alpha=A_{22}-A_{21} A_{11}^{-1} A_{12}=\left(a_{s t}^{\prime}\right) .
$$

Then by Theorem 1 in [14], we have:

$$
\left|a_{t t}^{\prime}\right|-r_{t}(A / \alpha) \geq\left|a_{j_{t} j_{t}}\right|-\sum_{\substack{k \in[m] \\ k \neq t}}\left|a_{j j_{j}}\right|-w_{j_{t}}(A) .
$$


Applying the Varah's bound to $A / \alpha$, we get:

$$
\left\|(A / \alpha)^{-1}\right\|_{\infty} \leq \max _{j \in \alpha^{\prime}} \frac{1}{\left|a_{j j}\right|-\sum_{\substack{k \in \alpha^{\prime} \\ k \neq j}}\left|a_{j k}\right|-w_{j}(A)},
$$

where

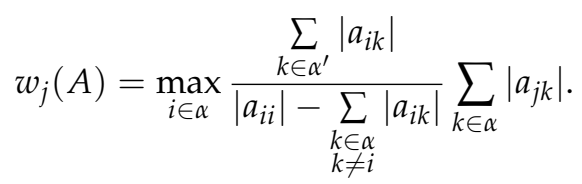

By Theorem 3,

$$
\left\|A_{11}^{-1}\right\|_{\infty} \leq \max \left\{\max _{\substack{i \in N_{1} \cap \alpha \\ j \in N_{2} \cap \alpha}} \frac{\left|a_{j j}\right|-\beta_{j}+\beta_{i}}{\left(\left|a_{i i}\right|-\alpha_{i}\right)\left(\left|a_{j j}\right|-\beta_{j}\right)-\beta_{i} \alpha_{j}}, \max _{\substack{i \in N_{1} \wedge \alpha \\ j \in N_{2} \cap \alpha}} \frac{\left|a_{i i}\right|-\alpha_{i}+\alpha_{j}}{\left(\left|a_{i i}\right|-\alpha_{i}\right)\left(\left|a_{j j}\right|-\beta_{j}\right)-\beta_{i} \alpha_{j}}\right\} .
$$

From (7), (9), (46), (47), and Corollary 1, the conclusion follows.

Theorem 8. Let $A=\left[a_{i j}\right]$ be GDSDD $D_{n}^{N_{1}, N_{2}}$ with $a_{i i} \neq 0, i \in N$, and $\varnothing \neq \alpha \subset N$. Assume that (8) holds, if $N_{1}=\alpha$ or $N_{2}=\alpha$, then:

$$
\left\|A^{-1}\right\|_{\infty} \leq \gamma(\alpha) \cdot\left(1+\max _{i \in \alpha} \frac{R_{i}\left(A_{12}\right)}{\left|a_{i i}\right|-r_{i}\left(A_{11}\right)}\right) \cdot \max \left\{\max _{i \in \alpha} \frac{1}{\left|a_{i i}\right|-r_{i}\left(A_{11}\right)}, \max _{j \in \alpha^{\prime}} \frac{1}{\left|a_{j j}\right|-\sum_{\substack{k \in \alpha^{\prime} \\ k \neq j}}\left|a_{j k}\right|-w_{j}(A)}\right\},
$$

where

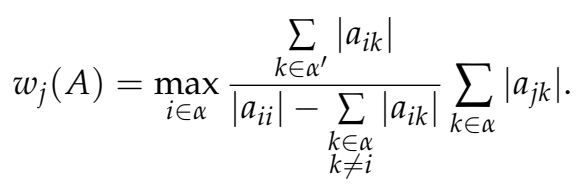

Proof. Since $A$ is $G D S D D_{n}^{N_{1}, N_{2}}, N_{1}=\alpha$ or $N_{2}=\alpha$, by Lemmas 5 and 6 , we have $A_{11}$ and $A / \alpha$ is $S D D$, consequently, nonsingular. Applying the Varah's bound to $A_{11}$, we get:

$$
\left\|A_{11}^{-1}\right\|_{\infty} \leq \max _{i \in \alpha} \frac{1}{\left|a_{i i}\right|-r_{i}\left(A_{11}\right)} .
$$

From Corollary 1, we can obtain:

$$
\|Q\|_{\infty} \leq 1+\max _{i \in \alpha} \frac{R_{i}\left(A_{12}\right)}{\left|a_{i i}\right|-r_{i}\left(A_{11}\right)} .
$$

Let,

$$
A / \alpha=A_{22}-A_{21} A_{11}^{-1} A_{12}=\left(a_{s t}^{\prime}\right) .
$$

Then by Theorem 1 in [14], we have:

$$
\left|a_{t t}^{\prime}\right|-r_{t}(A / \alpha) \geq\left|a_{j_{t} j_{t}}\right|-\sum_{\substack{k \in[m] \\ k \neq t}}\left|a_{j_{t} j_{k}}\right|-w_{j_{t}}(A) .
$$


Applying the Varah's bound to $A / \alpha$, we get:

$$
\left\|(A / \alpha)^{-1}\right\|_{\infty} \leq \max _{j \in \alpha^{\prime}} \frac{1}{\left|a_{j j}\right|-\sum_{\substack{k \in \alpha^{\prime} \\ k \neq j}}\left|a_{j k}\right|-w_{j}(A)},
$$

where

$$
w_{j}(A)=\max _{i \in \alpha} \frac{\sum_{k \in \alpha^{\prime}}\left|a_{i k}\right|}{\left|a_{i i}\right|-\sum_{\substack{k \in \alpha \\ k \neq i}}\left|a_{i k}\right|} \sum_{k \in \alpha}\left|a_{j k}\right| .
$$

From (7), (9), (48)-(50), the conclusion follows.

Theorem 9. Let $A=\left[a_{i j}\right]$ be GDSDD $D_{n}^{N_{1}, N_{2}}$, and $n \geq 3$. Then:

$$
\left\|A^{-1}\right\|_{\infty} \leq \Delta(A)=\min _{i \in N} \Delta_{i}(A),
$$

where

$$
\begin{aligned}
& \Delta_{i}(A)=\left(1+\frac{\max _{j \in N, j \neq i}\left|a_{j i}\right|}{\left|a_{i i}\right|}\right)\left(1+\frac{\max _{j \in N, j \neq i}\left|a_{i j}\right|}{\left|a_{i i}\right|}\right) \tilde{\Delta}_{i}(A), \\
& \tilde{\Delta}_{i}(A)=\max \left\{\frac{1}{\left|a_{i i}\right|}, \Delta^{\prime}(A)\right\}, \\
& \Delta^{\prime}(A)=\max \left\{\max _{\substack{j \in N_{1}-\{i\} \\
k \in N_{2}-\{i\}}} \frac{\left|c_{k k}\right|-\sum_{\substack{p \in N_{2} \\
p \neq k, i}}\left|c_{k p}\right|+\sum_{p \in N_{2}, p \neq i}\left|c_{j p}\right|}{\left(\left|c_{j j}\right|-\sum_{\substack{p \in N_{1} \\
p \neq j, i}}\left|c_{j p}\right|\right)\left(\left|c_{k k}\right|-\sum_{\substack{p \in N_{2} \\
p \neq k, i}}\left|c_{k p}\right|\right)-\sum_{p \in N_{1}, p \neq i}\left|c_{k p}\right| \sum_{p \in N_{2}, p \neq i}\left|c_{j p}\right|},\right. \\
& \left|c_{j j}\right|-\sum_{p \in N_{1}}\left|c_{j p}\right|+\sum_{p \in N_{1}, p \neq i}\left|c_{k p}\right| \\
& \left.\max _{\substack{j \in N_{1}-\{i\} \\
k \in N_{2}-\{i\}}} \frac{\substack{p \in N_{1} \\
p \neq j, i}}{\left(\left|c_{j j}\right|-\sum_{\substack{p \in N_{1} \\
p \neq j, i}}\left|c_{j p}\right|\right)\left(\left|c_{k k}\right|-\sum_{\substack{p \in N_{2} \\
p \neq k, i}}\left|c_{k p}\right|\right)-\sum_{p \in N_{1}, p \neq i}\left|c_{k p}\right| \sum_{p \in N_{2}, p \neq i}\left|c_{j p}\right|}\right\} \text {, }
\end{aligned}
$$

and

$$
c_{j k}=a_{j k}-\frac{a_{j i} a_{i k}}{a_{i i}}, j, k \in N-\{i\} .
$$

Proof. Since $A$ is GDSDD, then for any $\varnothing \neq \alpha \subset N$, by Lemmas 5 and 6 , we have $A_{11}$ is $G D S D D_{|\alpha|}^{N_{1} \cap \alpha, N_{2} \cap \alpha}, A / \alpha$ is $G D S D D_{|N-\alpha|}^{N_{1}-\alpha, N_{2}-\alpha}$, and consequently, they are nonsingular. Taking $\alpha=\{i\}$, then $A_{11}=a_{i i}, \alpha^{\prime}=N-\{i\}$ and:

$$
[A(\alpha)]^{-1}=\frac{1}{a_{i i}} \text {. }
$$

We have:

$$
\|S\|_{\infty}=1+\frac{\max _{s \in[m]}\left|a_{j_{s}}\right|}{\left|a_{i i}\right|}=1+\frac{\max _{j \in N, j \neq i}\left|a_{j i}\right|}{\left|a_{i i}\right|},
$$


and

$$
\|Q\|_{\infty}=1+\frac{\max _{s \in[m]}\left|a_{i j_{s}}\right|}{\left|a_{i i}\right|}=1+\frac{\max _{j \in N, j \neq i}\left|a_{i j}\right|}{\left|a_{i i}\right|} .
$$

Let $A / \alpha=\left(a_{s t}^{\prime}\right)$. By (22), we have:

$$
\left|a_{s t}^{\prime}\right|=\left|a_{j_{s} j_{t}}-\frac{a_{j_{s}} a_{i j_{t}}}{a_{i i}}\right|=\left|c_{j_{s} j_{t}}\right|, s, t \in[m], j_{s}, j_{t} \in N-\{i\} .
$$

By calculation, we obtain for $j_{s} \in N_{1}-\{i\}, j_{t} \in N_{2}-\{i\}$,

$$
\begin{aligned}
& \alpha_{s}(A / \alpha)=\sum_{\substack{j_{p} \in N_{1}-\{i\} \\
p \neq s}}\left|a_{j_{s} j_{p}}-\frac{a_{j_{s}} a_{i j_{p}}}{a_{i i}}\right|=\sum_{\substack{j_{p} \in N_{1} \\
j_{p} \neq j_{s}, i}}\left|c_{j_{s} j_{p}}\right|, \\
& \beta_{S}(A / \alpha)=\sum_{j_{p} \in N_{2}-\{i\}}\left|a_{j_{s} j_{p}}-\frac{a_{j_{s} i} a_{i j_{p}}}{a_{i i}}\right|=\sum_{\substack{j_{p} \in N_{2} \\
j_{p} \neq i}}\left|c_{j_{s} j_{p}}\right|, \\
& \alpha_{t}(A / \alpha)=\sum_{j_{p} \in N_{1}-\{i\}}\left|a_{j_{t} j_{p}}-\frac{a_{j_{t} i} a_{i j_{p}}}{a_{i i}}\right|=\sum_{\substack{j_{p} \in N_{1} \\
j_{p} \neq i}}\left|c_{j_{t} j_{p}}\right|,
\end{aligned}
$$

and

$$
\beta_{t}(A / \alpha)=\sum_{\substack{j_{p} \in N_{2}-\{i\} \\ p \neq t}}\left|a_{j_{t} j_{p}}-\frac{a_{j_{t}} a_{i j_{p}}}{a_{i i}}\right|=\sum_{\substack{j_{p} \in N_{2} \\ j_{p} \neq j, i}}\left|c_{j_{t} j_{p}}\right| .
$$

By (40), we have:

$$
\begin{aligned}
&\left\|(A / \alpha)^{-1}\right\|_{\infty} \leq \max \left\{\max _{\substack{j \in N_{1}-\{i\} \\
k \in N_{2}-\{i\}}} \frac{\left|c_{k k}\right|-\sum_{\substack{p \in N_{2} \\
p \neq k, i}}\left|c_{k p}\right|+\sum_{p \in N_{2}, p \neq i}\left|c_{j p}\right|}{\left(c_{j j}\left|-\sum_{\substack{p \in N_{1} \\
p \neq j, i}}\right| c_{j p} \mid\right)\left(\left|c_{k k}\right|-\sum_{\substack{p \in N_{2} \\
p \neq k, i}}\left|c_{k p}\right|\right)-\sum_{p \in N_{1}, p \neq i}\left|c_{k p}\right| \sum_{p \in N_{2}, p \neq i}\left|c_{j p}\right|},\right. \\
&\left.\max _{\substack{j \in N_{1}-\{i\} \\
k \in N_{2}-\{i\}}} \frac{\left|c_{j j}\right|-\sum_{\substack{p \in N_{1} \\
p \neq j, i}}\left|c_{j p}\right|+\sum_{p \in N_{1}, p \neq i}\left|c_{k p}\right|}{\left(c_{j j}\left|-\sum_{\substack{p \in N_{1} \\
p \neq j, i}}\right| c_{j p} \mid\right)\left(\left|c_{k k}\right|-\sum_{\substack{p \in N_{2} \\
p \neq k, i}}\left|c_{k p}\right|\right)-\sum_{p \in N_{1}, p \neq i}\left|c_{k p}\right| \sum_{p \in N_{2}, p \neq i}\left|c_{j p}\right|}\right\} .
\end{aligned}
$$

By (7), (51)-(54), we have $\left\|A^{-1}\right\|_{\infty} \leq \Delta_{i}(A)$. By the arbitrariness of $i$, the conclusion follows.

In the following, we prove that the bound in Theorem 9 generally improves the bound obtained by Theorem 3 in [11] for SDD matrices and Theorem 9 in [12] for DSDD matrices.

Theorem 10. Let $A=\left[a_{i j}\right] \in C^{n \times n}$ be SDD. Then,

$$
\left\|A^{-1}\right\|_{\infty} \leq \Delta(A) \leq B_{\text {Schur }}(A)=\min _{i \in N} B_{\text {Schur }}(i),
$$

where $\Delta(A)$ is defined in Theorem 9 and: 


$$
B_{\text {Schur }}(i)=2\left(1+\frac{\max _{j \in N, j \neq i}\left|a_{j i}\right|}{\left|a_{i i}\right|}\right) \max \left\{\frac{1}{\left|a_{i i}\right|}, \max _{j \in N, j \neq i} \frac{1}{\left|a_{j j}\right|-r_{j}(A)+\frac{\left|a_{i i}\right|-r_{i}(A)}{\left|a_{i i}\right|}\left|a_{j i}\right|}\right\} .
$$

Proof. Since $A$ is $S D D, A$ is GDSDD with $N_{1}=N, N_{2}=\varnothing$. From Theorem 9, we can obtain:

$$
\left\|A^{-1}\right\|_{\infty} \leq \Delta(A)=\min _{i \in N} \Delta_{i}(A),
$$

where

$$
\begin{gathered}
\Delta_{i}(A)=\left(1+\frac{\max _{j \in N, j \neq i}\left|a_{j i}\right|}{\left|a_{i i}\right|}\right)\left(1+\frac{\max _{j \in N, j \neq i}\left|a_{i j}\right|}{\left|a_{i i}\right|}\right) \tilde{\Delta}_{i}(A), \\
\tilde{\Delta}_{i}(A)=\max \left\{\frac{1}{\left|a_{i i}\right|}, \Delta^{\prime}(A)\right\}, \\
\Delta^{\prime}(A)=\max _{j \in N, j \neq i} \frac{1}{\left|c_{j j}\right|-\sum_{p \in N, p \neq j, i}\left|c_{j p}\right|^{\prime}}
\end{gathered}
$$

and

$$
c_{j k}=a_{j k}-\frac{a_{j i} a_{i k}}{a_{i i}}, j, k \in N-\{i\} .
$$

From Theorem 3 of [11],

$$
\left\|A^{-1}\right\|_{\infty} \leq B_{\text {Schur }}(A)=\min _{i \in N} B_{\text {Schur }}(i),
$$

where

$$
B_{\text {Schur }}(i)=2\left(1+\frac{\max _{j \in N, j \neq i}\left|a_{j i}\right|}{\left|a_{i i}\right|}\right) \max \left\{\frac{1}{\left|a_{i i}\right|} \max _{j \in N, j \neq i} \frac{1}{\left|a_{j j}\right|-r_{j}(A)+\frac{\left|a_{i i}\right|-r_{i}(A)}{\left|a_{i i}\right|}\left|a_{j i}\right|}\right\} .
$$

By calculation,

$$
\begin{aligned}
\left|c_{j j}\right|-\sum_{p \in N, p \neq j, i}\left|c_{j p}\right| & =\left|a_{j j}-\frac{a_{j i} a_{i j}}{a_{i i}}\right|-\sum_{p \in N, p \neq j, i}\left|a_{j p}-\frac{a_{j i} a_{i p}}{a_{i i}}\right| \\
& \geq\left|a_{j j}\right|-\frac{\left|a_{j i}\right|\left|a_{i j}\right|}{\left|a_{i i}\right|}-\sum_{p \in N, j \neq j, i}\left(\left|a_{j p}\right|+\frac{\left|a_{j i}\right|\left|a_{i p}\right|}{\left|a_{i i}\right|}\right) \\
& =\left|a_{j j}\right|-\frac{\left|a_{j i}\right|\left|a_{i j}\right|}{\left|a_{i i}\right|}-\left(\sum_{p \in N, j \neq j, i}\left|a_{j p}\right|+\sum_{p \in N, p \neq j, i} \frac{\left|a_{j i}\right|\left|a_{i p}\right|}{\left|a_{i i}\right|}\right) \\
& =\left|a_{j j}\right|-\sum_{p \in N, j \neq j, i}\left|a_{j p}\right|-\frac{\left|a_{j i}\right|\left|a_{i j}\right|}{\left|a_{i i}\right|}-\sum_{p \in N, p \neq j, i} \frac{\left|a_{j i}\right|\left|a_{i p}\right|}{\left|a_{i i}\right|} \\
& =\left|a_{j j}\right|-\sum_{p \in N, j \neq j, i}\left|a_{j p}\right|-\left|a_{j i}\right|+\left|a_{j i}\right|\left(1-\frac{\left|a_{i j}\right|}{\left|a_{i i}\right|}-\sum_{p \in N, p \neq j, i} \frac{\left|a_{i p}\right|}{\left|a_{i i}\right|}\right) \\
& =\left|a_{j j}\right|-r_{j}(A)+\left|a_{j i}\right|\left(1-\frac{r_{i}(A)}{\left|a_{i i}\right|}\right) \\
& =\left|a_{j j}\right|-r_{j}(A)+\frac{\left|a_{i i}\right|-r_{i}(A)}{\left|a_{i i}\right|}\left|a_{j i}\right| .
\end{aligned}
$$


Then,

$$
\max _{j \in N, j \neq i} \frac{1}{\left|c_{j j}\right|-\sum_{p \in N, p \neq j, i}\left|c_{j p}\right|} \leq \max _{j \in N, j \neq i} \frac{1}{\left|a_{j j}\right|-r_{j}(A)+\frac{\left|a_{i i}\right|-r_{i}(A)}{\left|a_{i i}\right|}\left|a_{j i}\right|} .
$$

Since $A$ is $S D D$,

$$
\left|a_{i i}\right|>r_{i}(A)=\sum_{j \in N, j \neq i}\left|a_{i j}\right|, i \in N
$$

For all $i \in N$, it is easy to get:

$$
\begin{gathered}
\frac{\sum_{j \in N, j \neq i}\left|a_{i j}\right|}{\left|a_{i i}\right|}<1, \\
\left(1+\frac{\max _{j \in N, j \neq i}\left|a_{i j}\right|}{\left|a_{i i}\right|}\right)<2 .
\end{gathered}
$$

Therefore,

$$
\Delta_{i}(A)<B_{\text {Schur }}(i), i \in N .
$$

Furthermore,

$$
\Delta(A)<B_{\text {Schur }}(A) .
$$

The proof is completed.

Theorem 10 shows that the bound in Theorem 9 generally improves the bound obtained by Theorem 3 in [11] for SDD matrices.

Theorem 11. Let $A=\left[a_{i j}\right] \in C^{n \times n}$ be DSDD. Then:

$$
\left\|A^{-1}\right\|_{\infty} \leq \Delta(A) \leq \Pi(A)=\min _{i \in N} \pi_{i}(A),
$$

where $\Delta(A)$ is defined in Theorem 9 ,

$$
\begin{gathered}
\pi_{i}(A)=\left(1+\frac{\max _{i \in N, j \neq i}\left|a_{j i}\right|}{\left|a_{i i}\right|}\right)\left(1+\frac{\max _{j \in N, j \neq i}\left|a_{i j}\right|}{\left|a_{i i}\right|}\right) \tilde{\pi}_{i}(A), \\
\tilde{\pi}_{i}(A)=\max \left\{\frac{1}{\left|a_{i i}\right|}, \max _{j, k \in N-\{i\}} \frac{\left|c_{k k}\right|+\sum_{j \in N, p \neq i, j}\left|c_{j p}\right|}{\left|c_{j j}\right|\left|c_{k k}\right|-\sum_{p \in N, p \neq i, j}\left|c_{j p}\right| \sum_{p \in N, p \neq i, k}\left|c_{k p}\right|}\right\},
\end{gathered}
$$

and

$$
c_{j k}=a_{j k}-\frac{a_{j i} a_{i k}}{a_{i i}}, j, k \in N-\{i\} .
$$

Proof. Since $A$ is $D S D D$, there is at most one $i_{0}$ such that $\left|a_{i_{0} i_{0}}\right| \leq r_{i_{0}}(A)$. If there does not exist an $i_{0}$ such that $\left|a_{i_{0} i_{0}}\right| \leq r_{i_{0}}(A)$, then $A$ is $S D D$, the proof is the same as Theorem 10. If there exists only one $i_{0}$ such that $\left|a_{i_{0} i_{0}}\right| \leq r_{i_{0}}(A)$, then $A$ is GDSDD with $N_{1}=N-\left\{i_{0}\right\}, N_{2}=\left\{i_{0}\right\}$. From Theorem 9, we can obtain: 


$$
\left\|A^{-1}\right\|_{\infty} \leq \Delta(A)=\min _{i \in N} \Delta_{i}(A),
$$

where

$$
\begin{aligned}
& \Delta_{i}(A)=\left(1+\frac{\max _{j \in N, j \neq i}\left|a_{j i}\right|}{\left|a_{i i}\right|}\right)\left(1+\frac{\max _{j \in N, j \neq i}\left|a_{i j}\right|}{\left|a_{i i}\right|}\right) \tilde{\Delta}_{i}(A) \\
& \tilde{\Delta}_{i}(A)=\max \left\{\frac{1}{\left|a_{i i}\right|}, \Delta^{\prime}(A)\right\}, \\
& \Delta^{\prime}(A)=\max \left\{\max _{\substack{j \in N-\left\{i_{0}\right\}-\{i\} \\
k \in\left\{i_{0}\right\}-\{i\}}} \frac{\left|c_{k k}\right|+\sum_{p \in\left\{i_{0}\right\}, p \neq i}\left|c_{j p}\right|}{\left(\left|c_{j j}\right|-\sum_{p \in N-\left\{i_{0}\right\}, p \neq j, i}\left|c_{j p}\right|\right)\left|c_{k k}\right|-\sum_{p \in N-\left\{i_{0}\right\}, p \neq i}\left|c_{k p}\right| \sum_{p \in\left\{i_{0}\right\}, p \neq i}\left|c_{j p}\right|^{\prime}},\right. \\
& \left.\frac{\left|c_{j j}\right|-\sum_{p \in N-\left\{i_{0}\right\}, p \neq j, i}\left|c_{j p}\right|+\sum_{p \in N-\left\{i_{0}\right\}, p \neq i}\left|c_{k p}\right|}{\left.\sum_{p \in N-\left\{i_{0}\right\}, p \neq j, i}\left|c_{j p}\right|\right)\left|c_{k k}\right|-\sum_{p \in N-\left\{i_{0}\right\}, p \neq i}\left|c_{k p}\right| \sum_{p \in\left\{i_{0}\right\}, p \neq i}\left|c_{j p}\right|}\right\} \text {. }
\end{aligned}
$$

From Theorem 9 of [12],

$$
\left\|A^{-1}\right\|_{\infty} \leq \Pi(A)=\min _{i \in N} \pi_{i}(A),
$$

where

$$
\begin{aligned}
& \pi_{i}(A)=\left(1+\frac{\max _{i \in N, j \neq i}\left|a_{j i}\right|}{\left|a_{i i}\right|}\right)\left(1+\frac{\max _{j \in N, j \neq i}\left|a_{i j}\right|}{\left|a_{i i}\right|}\right) \tilde{\pi}_{i}(A),
\end{aligned}
$$

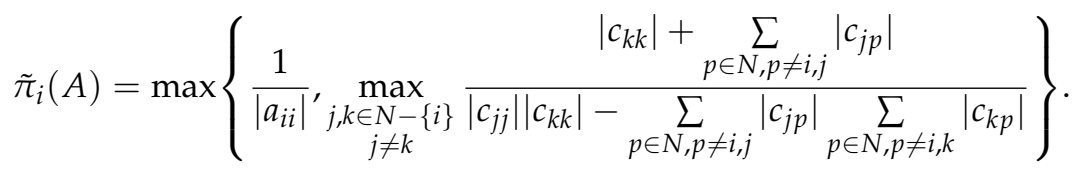

For $i \neq i_{0}$,

$$
\begin{aligned}
\Delta^{\prime}(A)=\max & \left\{\max _{j \in N-\left\{i_{0}\right\}-\{i\}} \frac{\left|c_{i_{0} i_{0}}\right|+\left|c_{j i_{0}}\right|}{\left|c_{j j}\right|\left|c_{i_{0} i_{0}}\right|-\left|c_{i_{0} i_{0}}\right| \sum_{p \in N-\left\{i_{0}\right\}, p \neq j, i}\left|c_{j p}\right|-\left|c_{j i_{0}}\right| \sum_{p \in N-\left\{i_{0}\right\}, p \neq i}\left|c_{i_{0} p}\right|},\right. \\
& \left.\max _{\substack{j \in N-\left\{i_{0}\right\}-\{i\} \\
k=i_{0}}} \frac{\left|c_{j j}\right|-\sum_{p \in N-\left\{i_{0}\right\}, p \neq j, i}\left|c_{j p}\right|+\sum_{p \in N-\left\{i_{0}\right\}, p \neq i}\left|c_{i_{0} p}\right|}{\left|c_{j j}\right|\left|c_{i_{0} i_{0}}\right|-\left|c_{i_{0} i_{0}}\right| \sum_{p \in N-\left\{i_{0}\right\}, p \neq j, i}\left|c_{j p}\right|-\left|c_{j i_{0}}\right| \sum_{p \in N-\left\{i_{0}\right\}, p \neq i}\left|c_{i_{0} p}\right|}\right\} .
\end{aligned}
$$

Obviously,

$$
\begin{aligned}
& \max _{\substack{j, k \in N-\{i\} \\
j \neq k}} \frac{\left|c_{k k}\right|+\sum_{p \in N, p \neq i, j}\left|c_{j p}\right|}{\left|c_{j j}\right|\left|c_{k k}\right|-\sum_{p \in N, p \neq i, j}\left|c_{j p}\right| \sum_{p \in N, p \neq i, k}\left|c_{k p}\right|} \\
& \geq \max _{\substack{j \in N-\left\{i_{0}\right\}-\{i\} \\
k=i_{0}}} \frac{\left|c_{i_{0} i_{0}}\right|+\sum_{p \in N, p \neq i, j}\left|c_{j p}\right|}{\left|c_{j j}\right|\left|c_{i_{0} i_{0}}\right|-\sum_{p \in N, p \neq i, j}\left|c_{j p}\right| \sum_{p \in N, p \neq i, i_{0}}\left|c_{i_{0} p}\right|}
\end{aligned}
$$




$$
\begin{gathered}
\max _{\substack{j, k \in N-\{i\} \\
j \neq k}} \frac{\left|c_{k k}\right|+\sum_{p \in N, p \neq i, j}\left|c_{j p}\right|}{\left|c_{j j}\right|\left|c_{k k}\right|-\sum_{p \in N, p \neq i, j}\left|c_{j p}\right| \sum_{p \in N, p \neq i, k}\left|c_{k p}\right|} \\
\geq \max _{\substack{j=i_{0} \\
k \in N-\left\{i_{0}\right\}-\{i\}}} \frac{\left|c_{k k}\right|+\sum_{p \in N, p \neq i, i_{0}}\left|c_{i_{0} p}\right|}{\left|c_{i_{0} i_{0}}\right|\left|c_{k k}\right|-\sum_{p \in N, p \neq i, i_{0}}\left|c_{i_{0}} p\right| \sum_{p \in N, p \neq i, k}\left|c_{k p}\right|}
\end{gathered}
$$

\section{We can obtain:}

$$
\left|c_{i_{0} i_{0}}\right|+\left|c_{j i_{0}}\right| \leq\left|c_{i_{0} i_{0}}\right|+\sum_{p \in N, p \neq i, j}\left|c_{j p}\right|,
$$

$$
\begin{aligned}
\left|c_{j j}\right|\left|c_{i_{0} i_{0}}\right|-\sum_{p \in N, p \neq i, j}\left|c_{j p}\right| \sum_{p \in N, p \neq i, i_{0}}\left|c_{i_{0} p}\right| & =\left|c_{j j}\right|\left|c_{i_{0} i_{0}}\right|-\left(\left|c_{j i_{0}}\right|+\sum_{p \in N, p \neq i, j, i_{0}}\left|c_{j p}\right|\right) \sum_{p \in N, p \neq i, i_{0}}\left|c_{i_{0} p}\right| \\
& =\left|c_{j j}\right|\left|c_{i_{0} i_{0}}\right|-\sum_{p \in N, p \neq i, j, i_{0}}\left|c_{j p}\right| \sum_{p \in N, p \neq i, i_{0}}\left|c_{i_{0} p}\right|-\left|c_{j i_{0}}\right| \sum_{p \in N, p \neq i, i_{0}}\left|c_{i_{0} p}\right| \\
& \leq\left|c_{j j}\right|\left|c_{i_{0} i_{0}}\right|-\left|c_{i_{0} i_{0}}\right| \sum_{p \in N, p \neq i, j, i_{0}}\left|c_{j p}\right|-\left|c_{j i_{0}}\right| \sum_{p \in N, p \neq i, i_{0}}\left|c_{i_{0} p}\right| \\
& =\left|c_{j j}\right|\left|c_{i_{0} i_{0}}\right|-\left|c_{i_{0} i_{0}}\right| \sum_{p \in N-\left\{i_{0}\right\}, p \neq i, j}\left|c_{j p}\right|-\left|c_{j i_{0}}\right| \sum_{p \in N-\left\{i_{0}\right\}, p \neq i}\left|c_{i_{0} p}\right| .
\end{aligned}
$$

\section{Therefore,}

$$
\begin{aligned}
& \max _{\substack{j \in N-\left\{i_{0}\right\}-\{i\} \\
k=i_{0}}} \frac{\left|c_{i_{0} i_{0}}\right|+\left|c_{j i_{0}}\right|}{\left|c_{j j}\right|\left|c_{i_{0} i_{0}}\right|-\left|c_{i_{0} i_{0}}\right| \sum_{p \in N-\left\{i_{0}\right\}, p \neq j, i}\left|c_{j p}\right|-\left|c_{j i_{0}}\right| \sum_{p \in N-\left\{i_{0}\right\}, p \neq i}\left|c_{i_{0} p}\right|} \\
& \leq \max _{\substack{j \in N-\left\{i_{0}\right\}-\{i\} \\
k=i_{0}}} \frac{\left|c_{i_{0} i_{0}}\right|+\sum_{p \in N, p \neq i, j}\left|c_{j p}\right|}{\left|c_{j j}\right|\left|c_{i_{0} i_{0}}\right|-\sum_{p \in N, p \neq i, j}\left|c_{j p}\right| \sum_{p \in N, p \neq i, i_{0}}\left|c_{i_{0} p}\right|} .
\end{aligned}
$$

Similarly,

$$
\begin{aligned}
& \max _{\substack{j \in N-\left\{i_{0}\right\}-\{i\} \\
k=i_{0}}} \frac{\left|c_{j j}\right|-\sum_{p \in N-\left\{i_{0}\right\}, p \neq j, i}\left|c_{j p}\right|+\sum_{p \in N-\left\{i_{0}\right\}, p \neq i}\left|c_{i_{0} p}\right|}{\left(\left|c_{j j}\right|-\sum_{p \in N-\left\{i_{0}\right\}, p \neq j, i}\left|c_{j p}\right|\right)\left|c_{i_{0} i_{0}}\right|-\left|c_{j i_{0}}\right| \sum_{p \in N-\left\{i_{0}\right\}, p \neq i}\left|c_{i_{0} p}\right|} \\
& \leq \max _{\substack{j=i_{0} \\
k \in N-\left\{i_{0}\right\}-\{i\}}} \frac{\left|c_{k k}\right|+\sum_{p \in N, p \neq i, i_{0}}\left|c_{i_{0} p}\right|}{\left|c_{i_{0} i_{0}}\right|\left|c_{k k}\right|-\sum_{p \in N, p \neq i, i_{0}}\left|c_{i_{0} p}\right| \sum_{p \in N, p \neq i, k}\left|c_{k p}\right|} .
\end{aligned}
$$

Then,

$$
\begin{aligned}
& \max \left\{\max _{\substack{j \in N-\left\{i_{0}\right\}-\{i\} \\
k=i_{0}}} \frac{\left|c_{i_{0} i_{0}}\right|+\left|c_{j i_{0}}\right|}{\left(\left|c_{j j}\right|-\sum_{p \in N-\left\{i_{0}\right\}, p \neq j, i}\left|c_{j p}\right|\right)\left|c_{i_{0} i_{0}}\right|-\sum_{p \in N-\left\{i_{0}\right\}, p \neq i}\left|c_{i_{0} p}\right|\left|c_{j i_{0}}\right|},\right. \\
& \left.\max _{\substack{j \in N-\left\{i_{0}\right\}-\{i\} \\
k=i_{0}}} \frac{\left|c_{j j}\right|-\sum_{p \in N-\left\{i_{0}\right\}, p \neq j, i}\left|c_{j p}\right|+\sum_{p \in N-\left\{i_{0}\right\}, p \neq i}\left|c_{i_{0} p}\right|}{\left(\left|c_{j j}\right|-\sum_{p \in N-\left\{i_{0}\right\}, p \neq j, i}\left|c_{j p}\right|\right)\left|c_{i_{0} i_{0}}\right|-\sum_{p \in N-\left\{i_{0}\right\}, p \neq i}\left|c_{i_{0} p}\right|\left|c_{j i_{0}}\right|}\right\} \\
& \leq \max \left\{\max _{\substack{j \in N-\left\{i_{0}\right\}-\{i\} \\
k=i_{0}}} \frac{\left|c_{i_{0} i_{0}}\right|+\sum_{p \in N, p \neq i, j}\left|c_{j p}\right|}{\left(\left|c_{j j}\right|\left|c_{i_{0} i_{0}}\right|-\sum_{p \in N, p \neq i, j}\left|c_{j p}\right| \sum_{p \in N, p \neq i, i_{0}}\left|c_{i_{0} p}\right|\right.},\right.
\end{aligned}
$$




$$
\begin{aligned}
& \left.\max _{\substack{j=i_{0} \\
k \in N-\left\{i_{0}\right\}-\{i\}}} \frac{\left|c_{k k}\right|+\sum_{p \in N, p \neq i, i_{0}}\left|c_{i_{0} p}\right|}{\left|c_{i_{0} i_{0}}\right|\left|c_{k k}\right|-\sum_{p \in N, p \neq i, i_{0}}\left|c_{i_{0} p}\right| \sum_{p \in N, p \neq i, k}\left|c_{k p}\right|}\right\} \\
& \leq \max _{\substack{j, k \in N-\{i\} \\
j \neq k}} \frac{\left|c_{k k}\right|+\sum_{p \in N, p \neq i, j}\left|c_{j p}\right|}{\left|c_{j j}\right|\left|c_{k k}\right|-\sum_{p \in N, p \neq i, j}\left|c_{j p}\right| \sum_{p \in N, p \neq i, k}\left|c_{k p}\right|} .
\end{aligned}
$$

Therefore,

$$
\tilde{\Delta}_{i}(A) \leq \tilde{\pi}_{i}(A) .
$$

For $i=i_{0}$

$$
\begin{aligned}
& \tilde{\Delta}_{i_{0}}(A)=\frac{1}{\left|a_{i_{0} i_{0}}\right|},
\end{aligned}
$$

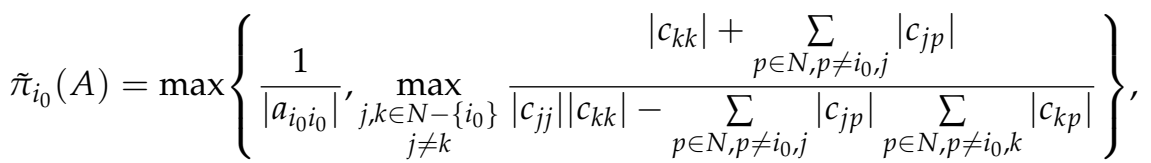

$$
\begin{aligned}
& \tilde{\Delta}_{i_{0}}(A) \leq \tilde{\pi}_{i_{0}}(A) .
\end{aligned}
$$

Furthermore,

$$
\begin{gathered}
\tilde{\Delta}_{i}(A) \leq \tilde{\pi}_{i}(A), i \in N, \\
\Delta_{i}(A) \leq \Pi_{i}(A), i \in N, \\
\Delta(A) \leq \Pi(A) .
\end{gathered}
$$

The proof is completed.

Theorem 11 shows that the bound in Theorem 9 generally improves the bound obtained by Theorem 9 in [12] for DSDD matrices.

We illustrate our results by the following two examples.

Example 1. Consider the bound for $\left\|A^{-1}\right\|_{\infty}$ of a SDD matrix $A$, where:

$$
A=\left[\begin{array}{cccc}
14 & 4 & 4 & 5 \\
1 & 19 & 4 & 5 \\
2 & 3 & 13 & 5 \\
4 & 5 & 4 & 14
\end{array}\right] .
$$

By Theorem 1, we have:

$$
\left\|A^{-1}\right\|_{\infty} \leq 1
$$

By Theorem 2, we have:

$$
\left\|A^{-1}\right\|_{\infty} \leq 1
$$


By Theorem 3, we have:

$$
\left\|A^{-1}\right\|_{\infty} \leq 1
$$

By Theorem 3 in [11], we have:

$$
\left\|A^{-1}\right\|_{\infty} \leq 0.8727
$$

By Theorem 9 in [12], we have:

$$
\left\|A^{-1}\right\|_{\infty} \leq 0.2200 .
$$

By Theorem 9, we have:

$$
\left\|A^{-1}\right\|_{\infty} \leq 0.2141
$$

In fact, $\left\|A^{-1}\right\|_{\infty}=0.1464$. This example shows that our bound is better than those in Theorems 1-3 and Theorem 3 in [11], Theorem 9.

Example 2. Consider the bound for $\left\|A^{-1}\right\|_{\infty}$ of a GDSDD matrix $A$ for $N_{1}=\{1,2\}$, $N_{2}=\{3,4\}$,

$$
\begin{gathered}
A=\left[\begin{array}{cccc}
12 & 5 & 1 & 1 \\
4 & 13 & 3 & 1 \\
2 & 0 & 3 & 2 \\
3 & 2 & 1 & 6
\end{array}\right], \\
A^{-1}=\left[\begin{array}{cccc}
0.0971 & -0.0354 & 0.0073 & -0.0127 \\
-0.0177 & 0.0812 & -0.0808 & 0.0163 \\
-0.0408 & 0.0336 & 0.3521 & -0.1162 \\
-0.0358 & -0.0150 & -0.0354 & 0.1869
\end{array}\right] .
\end{gathered}
$$

Note that:

$$
\left\|A^{-1}\right\|_{\infty}=0.5426
$$

We know that $A$ is neither a SDD matrix nor a DSDD matrix, and the bound of $\left\|A^{-1}\right\|_{\infty}$ can not be obtained by Theorems 1 and 2, Theorem 3 in [11], or Theorem 9 in [12], but it can be estimated by Theorems 3 and 9 in this paper.

By Theorem 3, we have:

$$
\left\|A^{-1}\right\|_{\infty} \leq 11
$$

The bound for $\left\|A^{-1}\right\|_{\infty}$ can be estimated by Theorem 9 as follows:

$$
\left\|A^{-1}\right\|_{\infty} \leq 1.0986
$$

\section{Applications to the Smallest Singular Value for GDSDD Matrices}

The singular values of an $n \times m$ complex matrix $A$ are the eigenvalues of $\sqrt{A A^{*}}$ and are denoted:

$$
\sigma_{1}(A) \geq \sigma_{2}(A) \geq \ldots \geq \sigma_{n}(A) \geq 0
$$

The smallest singular value plays a special role in expressing properties of matrices. It indicates not only whether $A$ is nonsingular, but also how far from the singular matrices 
$A$ is. In addition, the spectral condition number $\sigma_{1}(A) / \sigma_{n}(A)$ is important in studying numerical calculations involving $A[6]$. Hence, lower bounds for the smallest singular value of matrices are of interest.

For $S D D$ matrices case, Varah in [10] obtained a lower bound for $\sigma_{n}(A)$ which is listed as follows.

Theorem 12 ([10]). If a matrix $A=\left[a_{i j}\right] \in C^{n \times n}$ and its transpose $A^{\mathrm{T}}$ are all $S D D$, then:

$$
\sigma_{n}(A) \geq \sqrt{\min _{i \in N}\left\{\left|a_{i i}\right|-r_{i}(A)\right\} \cdot \min _{i \in N}\left\{\left|a_{i i}\right|-r_{i}\left(A^{\mathrm{T}}\right)\right\}} .
$$

Besides the lower bound in Theorem 12, there are other existing lower bounds for $\sigma_{n}(A)$, see $[5,6,11]$ and references therein. Based on Theorem 9, we can get a new lower bound for $\sigma_{n}(A)$.

Theorem 13. If a matrix $A=\left[a_{i j}\right] \in C^{n \times n}$ and its transpose $A^{\mathrm{T}}$ are all GDSDD, then:

$$
\sigma_{n}(A) \geq \sqrt{\frac{1}{\Delta(A) \cdot \Delta\left(A^{\mathrm{T}}\right)}}
$$

where $\Delta(A)$ is defined as in Theorem 9.

Proof. Since $A^{\mathrm{T}}$ is GDSDD, then:

$$
\left\|A^{-1}\right\|_{1}=\left\|\left(A^{-1}\right)^{\mathrm{T}}\right\|_{\infty}=\left\|\left(A^{\mathrm{T}}\right)^{-1}\right\|_{\infty} \leq \Delta\left(A^{\mathrm{T}}\right) .
$$

By the well-known inequality (see Theorem 2 in [5]):

$$
\left\|A^{-1}\right\|_{2}^{2} \leq\left\|A^{-1}\right\|_{1}\left\|A^{-1}\right\|_{\infty}
$$

we have:

$$
\left\|A^{-1}\right\|_{2}^{2} \leq \Delta\left(A^{\mathrm{T}}\right) \cdot \Delta(A),
$$

and hence:

$$
\left\|A^{-1}\right\|_{2}^{-1} \geq \sqrt{\frac{1}{\Delta(A) \cdot \Delta\left(A^{\mathrm{T}}\right)}} .
$$

By $\left\|A^{-1}\right\|_{2}^{-1}=\sigma_{n}(A)$, the conclusion follows.

\section{Conclusions}

Based on the fact that the Schur complement of a GDSDD matrix is also a GDSDD matrix, we in Theorems 4-8 presented an upper bound of $\left\|A^{-1}\right\|_{\infty}$ for a GDSDD matrix $A$ for any nonempty proper subset $\alpha$ of $N$. And then, for the case $|\alpha|=1$, we in Theorem 9 gave an upper bound for $\left\|A^{-1}\right\|_{\infty}$. Moreover, we show that the bound in Theorem 9 generally improves the bound obtained by Theorem 3 in [11] for SDD matrices and Theorem 9 in [12] for DSDD matrices.

Author Contributions: Y.L. and Y.W. contributed equally to this work. All authors have read and agreed to the published version of the manuscript.

Funding: This work is partly supported by the National Natural Science Foundations of China (31600299), Natural Science Basic Research Program of Shaanxi, China (2020JM-622), and the Postgraduate Innovative Research Project of Baoji University of Arts and Sciences (YJSCX20ZD05, YJSCX21YB10). 
Data Availability Statement: Not applicable.

Acknowledgments: The authors are grateful to the anonymous referee and Gao Lei for their valuable suggestions and comments.

Conflicts of Interest: The authors declare no conflict of interest.

\section{References}

1. Berman, A.; Plemmons, R.J. Nonnegative Matrices in the Mathematical Sciences; Academic Press: New York, NY, USA, 1979.

2. Gao, Y.M.; Wang, X.H. Criteria for generalized diagonally dominant matrices and M-matrices. Linear Algebra Its Appl. 1992, 169, 257-268. [CrossRef]

3. Horn, R.A.; Johnson, C.R. Matrix Analysis; Cambridge University Press: Cambridge, UK, 1985.

4. Cvetkovi, L. H-matrix theory vs. eigenvalue localization. Numer. Algorithms 2006, 42, 229-245. [CrossRef]

5. Huang, T.Z. Estimation of $\left\|A^{-1}\right\|_{\infty}$ and the smallest singular value. Comput. Math. Appl. 2008, 55, 1075-1080. [CrossRef]

6. Johnson, C.R. A Gersgorin-type lower bound for the smallest singular value. Linear Algebra Its Appl. 1989, 112, 1-7. [CrossRef]

7. Kosti, V.R.; Cvetkovi, L.; Cvetkovi, D.L. Pseudospectra localizations and their applications. Numer. Linear Algebra Appl. 2016, 23, 356-372. [CrossRef]

8. Li, C.; Cvetković, L.; Wei, Y.; Zhao, J. An infinity norm bound for the inverse of Dashnic-Zusmanovich type matrices with applications. Linear Algebra Its Appl. 2019, 565, 99-122. [CrossRef]

9. Liu, J.; Zhang, J.; Yu, L. The Schur complement of strictly doubly diagonally dominant matrices and its application. Linear Algebra Its Appl. 2012, 437, 168-183. [CrossRef]

10. Varah, J.M. A lower bound for the smallest singular value of a matrix. Linear Algebra Its Appl. 1975, 11, 3-5. [CrossRef]

11. Li, C.Q. Schur Complement-Based Infinity Norm Bounds for the Inverse of SDD Matrices. Bull. Malays. Math. Sci. Soc. 2020, 43, 3829-3845. [CrossRef]

12. Sang, C.L. Schur Complement-Based Infinity Norm Bounds for the Inverse of DSDD Matrices. Bull. Iran. Math. Soc. 2021, 47, 1379-1398. [CrossRef]

13. Moraa, N. Upper bounds for the infinity norm of the inverse of SDD and S-SDD matrices. J. Comput. Appl. Math. 2007, 206, 667-678.

14. Liu, J.; Huang, Y.; Zhang, F. The Schur complements of generalized doubly diagonally dominant matrices. Linear Algebra Its Appl. 2004, 378, 231-244. [CrossRef]

15. Liu, J.; Zhang, F. Disc Separation of the Schur Complement of Diagonally Dominant Matrices and Determinantal Bounds. SIAM J. Matrix Anal. Appl. 2005, 27, 665-674. [CrossRef]

16. Zhang, C.Y.; Xu, F.; Xu, Z.; Li, J. General H-matrices and their Schur complements. Front. Math. China 2014, 9, 1141-1168. [CrossRef]

17. Zhang, F.Z. The Schur Complement and Its Applications; Springer Science and Business Media: Berlin/Heidelberg, Germany, 2006.

18. Yong, X.R. Two properties of diagonally dominant matrices. Numer. Linear Algebra Appl. 1996, 3, 173-177. [CrossRef] 